Supplement of

\title{
Source apportionment of carbonaceous aerosols in Xi'an, China: insights from a full year of measurements of radiocarbon and the stable isotope ${ }^{13} \mathrm{C}$
}

Haiyan $\mathrm{Ni}^{1,2,3,4}$, Ru-Jin Huang ${ }^{2,3 *}$, Junji $\mathrm{Cao}^{3,5^{*}}$, Ting Zhang ${ }^{3}$, Meng Wang ${ }^{3}$, Harro A.J. Meijer ${ }^{1}$, Ulrike Dusek ${ }^{1}$

${ }^{1}$ Centre for Isotope Research (CIO), Energy and Sustainability Research Institute Groningen (ESRIG), University of Groningen, Groningen, 9747 AG, the Netherlands

${ }^{2}$ State Key Laboratory of Loess and Quaternary Geology (SKLLQG), Institute of Earth Environment, Chinese Academy of Sciences, Xi'an, 710061, China

${ }^{3}$ Key Laboratory of Aerosol Chemistry \& Physics (KLACP), Institute of Earth Environment, Chinese Academy of Sciences, Xi'an, 710061, China

${ }^{4}$ University of Chinese Academy of Sciences, Beijing, 100049, China

${ }^{5}$ Institute of Global Environmental Change, Xi'an Jiaotong University, Xi'an 710049, China

Correspondence to: Ru-Jin Huang (rujin.huang@ieecas.cn); Junji Cao (cao@loess.llqg.ac.cn) 


\section{S1 Sample selection for radiocarbon $\left({ }^{14} \mathrm{C}\right)$ measurements}

There are $58 \mathrm{PM}_{2.5}$ samples in total, with 13 collected in spring, 15 in summer, 12 in autumn, and 18 in winter. Six samples with varying $\mathrm{PM}_{2.5}$ mass and carbonaceous aerosols loading were selected per season for ${ }^{14} \mathrm{C}$ analysis. Air mass back trajectories (for identifying the probable sources and transport pathways of air pollutions) are also considered when selecting samples for ${ }^{14} \mathrm{C}$ analysis. $72 \mathrm{~h}$ air mass back trajectories starting $150 \mathrm{~m}$ above ground level at 2:00 UTC (10:00 a.m., local standard time) were calculated using NOAA HYSPLIT trajectory model. The best situation is that the back trajectories were similar between days with high $\mathrm{PM}_{2.5}$ loading and low-to-median $\mathrm{PM}_{2.5}$ loading, in which case, the influence of air pollution transport to the sampling site could be minimized. Back trajectories of selected samples are presented in Fig. S2.

\section{S2 Measurement of source markers (levoglucosan, hopanes, picene and water-soluble potassium)}

Organic makers including levoglucosan, picene and hopanes were quantified using the Gas chromatography-mass spectrometry (GC/MS) instrumentation. Filter pieces were extracted with a mixture of dichloromethane and methanol $(2: 1, \mathrm{v} / \mathrm{v})$ under ultrasonication. The extracts were concentrated using a rotary evaporator in vacuum and then blown down to dryness using a pure nitrogen stream. After reaction with N,O-bis-(trimethylsilyl) trifluoroacetamide (BSTFA) at $70{ }^{\circ} \mathrm{C}$ for $3 \mathrm{~h}$, the derivatives were determined using a GC/MS technique. GC/MS analysis of the derivatized fraction was performed using an Agilent 7890A GC coupled with an Agilent 5975 CMSD. The GC separation was carried out on a HP-5MS fused silica capillary column with the GC oven temperature programmed from $50{ }^{\circ} \mathrm{C}(2 \mathrm{~min})$ to $120^{\circ} \mathrm{C}$ at $15{ }^{\circ} \mathrm{C} \mathrm{min}{ }^{-1}$ and then to $300{ }^{\circ} \mathrm{C}$ at $5{ }^{\circ} \mathrm{C} \mathrm{min}{ }^{-1}$ with a final isothermal hold at $300{ }^{\circ} \mathrm{C}$ for $16 \mathrm{~min}$. The sample was injected in a splitless mode at an injector temperature of $280{ }^{\circ} \mathrm{C}$, and scanned from 50 to 650 Daltons using electron impact (EI) mode at $70 \mathrm{eV}$. GC/MS response factors were determined using authentic standards. We use the sum of measured hopanes (Ehopanes) in this study, including $17 \alpha(\mathrm{H}), 22,29,30$-Trisnorhopane, $17 \alpha(\mathrm{H}), 21 \beta(\mathrm{H})-30$ Norhopane, $\quad 17 \beta(\mathrm{H}), 21 \alpha(\mathrm{H})-30$-Norhopane, $\quad 17 \alpha(\mathrm{H}), 21 \beta(\mathrm{H})$-Hopane, $\quad 17 \alpha(\mathrm{H}), 21 \alpha(\mathrm{H})$-Hopane, $17 \beta(\mathrm{H}), 21 \alpha(\mathrm{H})$-Hopane, $17 \alpha(\mathrm{H}), 21 \beta(\mathrm{H})-(22 \mathrm{~S})$-Homohopane, and 17 $\alpha(\mathrm{H}), 21 \beta(\mathrm{H})-(22 \mathrm{R})$-Homohopane.

Water-soluble potassium $\left(\mathrm{K}^{+}\right)$was measured in water extracts using Ion Chromatography (Dionex 600, Thermal Scientific-Dionex, Sunnyvale, CA, USA). IonPac CS12A column was used for the separation of cations. $20 \mathrm{mM}$ methanesulfonic acid with a flow rate of $1 \mathrm{~mL} \mathrm{~min}^{-1}$ was utilized as eluent for cation separation. The MDLs for $\mathrm{K}^{+}$was $0.001 \mu \mathrm{g} \mathrm{mL}^{-1}$. Details of these measurements are described in $\mathrm{Li}$ et al. (2016a) and Zhang et al. (2011).

S3 Primary OC/EC ratios from biomass burning $\left(r_{\mathrm{bb}}\right)$, coal combustion $\left(r_{\text {coal }}\right)$, and liquid fossil fuel combustion ( $\left.\boldsymbol{r}_{\text {liq.fossil }}\right)$

There is considerable variability in the published OC/EC ratios for biomass burning (Fig. S14), coal combustion (Fig. S15) and liquid fossil fuel combustion (e.g., vehicle emissions; Table S9). OC/EC ratios differ due to variabilities in experimental factors, such as fuel types and properties, combustion 
conditions (e.g., smoldering vs. flaming), sampling and analysis methods (e.g., different protocols for OCEC measurements) etc.

When selecting OC/EC ratios for each source, we applied the following rules: first, we prioritize localized measurements of fresh emissions and estimations specific to China; second, OCEC measured by IMPROVE_A (Chow et al., 2007), the same protocol applied in this study, have higher priorities than those measured by other protocols. This is because different protocols (e.g., IMPROVE_A, IMPROVE and NIOSH) can lead to differences in OC/EC ratios up to over 3 times (Chow et al., 2001). The difference in OC/EC ratios between IMPROVE and IMPROVE_A can be up to a factor of 2 (Chow et al., 2007); third, for sources with limited data, average of the available data is used.

Biomass burning emissions are mixtures of emissions from crop residues open burning, crop residues burning in household stove, and wood burning in household stove etc. Higher OC/EC ratios are reported for crop residues open burning than those reported for similar fuels burned in household stove (Ni et al., 2015), perhaps due to a more complete combustion of household biofuels leading to higher $\mathrm{EC}$ emission. As it is difficult to estimate the distribution among different biomass burning subtype, here we take $\mathrm{OC} / \mathrm{EC}$ ratios from emission inventories, where major types of biomass burning are included. OC and EC emission amounts from previously reported emission inventories are summarized and their ratios are presented in Fig. S14. OC/EC ratios ranged from 3 to 7, with the mean of 4.4. This range covers the $\mathrm{OC} / \mathrm{EC}$ ratios from fresh emissions of wood combustion (e.g., 4.67 for wood burning in rural China by Shen et al. (2015) and crop residues burning (e.g., 7 for mixture of wheat straw, rice straw and corn stalk by Han et al. (2016). We took $\underline{\boldsymbol{r}_{\mathbf{b b}}=\mathbf{5}(\mathbf{3}-7)}$ to account for the variabilities of biomass burning emissions. A bit lower central value of 4.5 was used in previous ${ }^{14} \mathrm{C}$-based source apportionments (Zhang et al., 2014, 2015b).

$\mathrm{OC} / \mathrm{EC}$ ratios for fresh emissions from coal combustion are summarized in Fig. S15. A relatively wide range of OC/EC ratios is found, partially can be explained by different protocol applied to OC/EC measurements (Chow et al., 2001, 2004; Han et al., 2016). We took OC/EC ratios in the literature measured by IMPROVE_A protocol when available, to be consistent with our OCEC measurements. $1.4 \pm 1.3$ and $6.3 \pm 1.3$ (average \pm 1 standard deviation) are used as OC/EC ratios from bituminous and anthracite coal combustion, respectively. They are quantified from typical used coals in residential sector in China (Tian et al., 2017), and measured by IMPROVE_A protocol (Chow et al., 2007). The selected OC/EC ratios of $1.4 \pm 1.3$ and $6.3 \pm 1.3$ for bituminous and anthracite coal, respectively overlaps most of data in literatures (Fig. S15). The final OC/EC ratios for coal combustion $\left(r_{\text {coal }}\right)$ depends on the share of bituminous coal and anthracite coal. Bituminous and anthracite coal are apportioned $80 \%$ and $20 \%$ respectively, according to raw coal production data (Chen et al., 2005; Zhi et al., 2008), leading to $\underline{\underline{r}}_{\text {coal }}=2.38 \pm 0.44$ derived from Monte Carlo Simulation with assumption of triangular distribution. This ratio is similar to 2.25 derived from $\mathrm{OC}$ and $\mathrm{EC}$ emission amounts from emission inventories of coal combustion for 2000 , and 2.26 for the year 2005 estimated by Zhi et al. (2008).

Vehicle emissions can be influenced by vehicle type, fuel quality, speed of the vehicle, as well as the 
features of the road (He et al., 2008; Cheng et al., 2010; Cui et al., 2016). Literature searches were conducted (Table S9) to establish OC/EC ratios for vehicle emissions. Due to the limitation of published data, the lower/upper bonds were estimated as the mean of all lower/upper bounds from different datasets. The mean was then calculated as the average of the lower and upper bounds. The established $\underline{r}_{\text {liq.fossil }}$ ranged from 0.69 to 1.01 with the mean of 0.85 . 


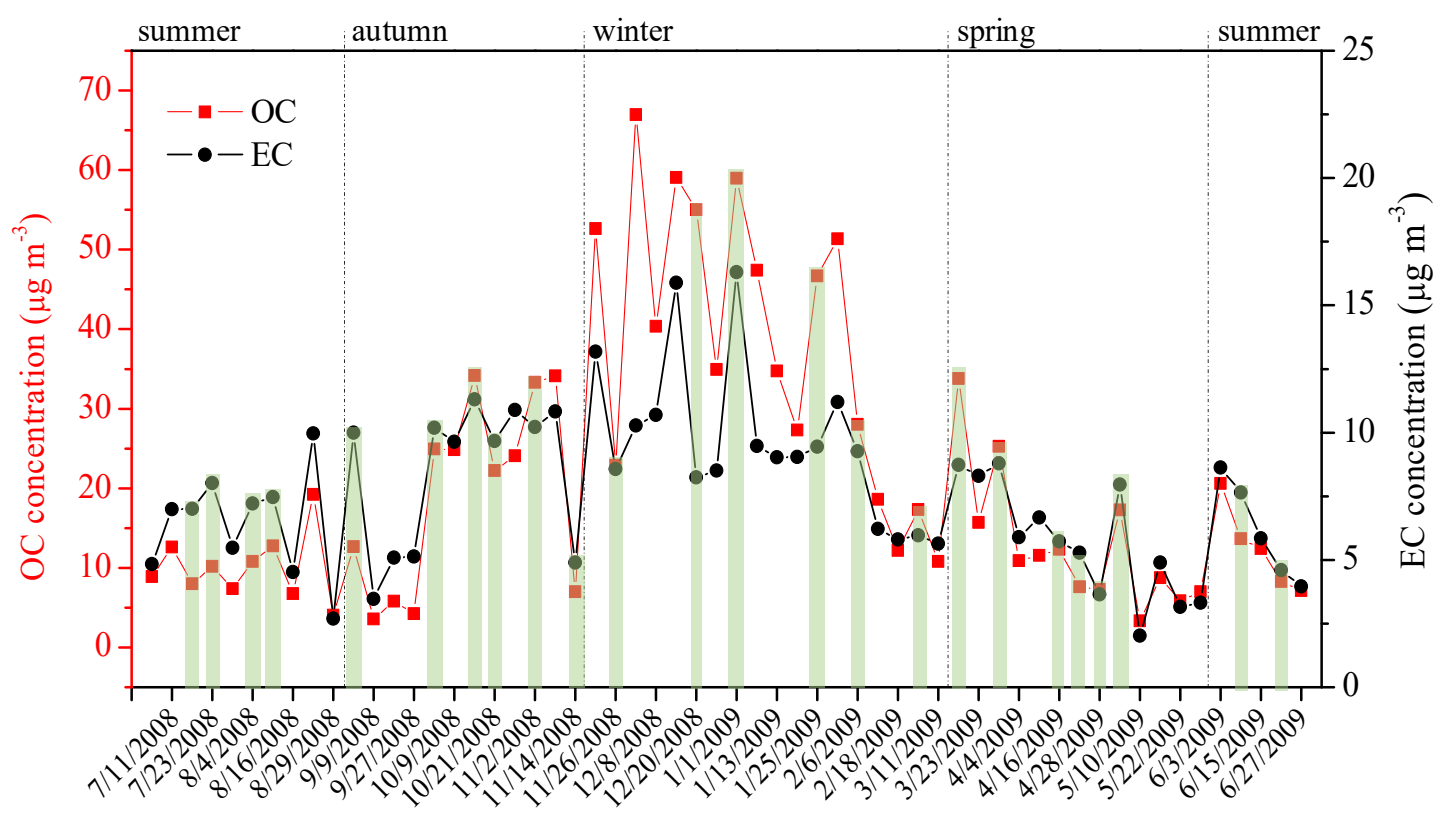

Figure S1. Temporal variability of OC and EC mass concentrations in PM2.5 in Xi'an, China, during July 2008 to June 2009 ( $n=58)$. Twenty-four samples were selected for ${ }^{14} \mathrm{C}$ analysis and highlighted in light green. Details on sample selection are presented in Supplemental S1. 
High PM loading
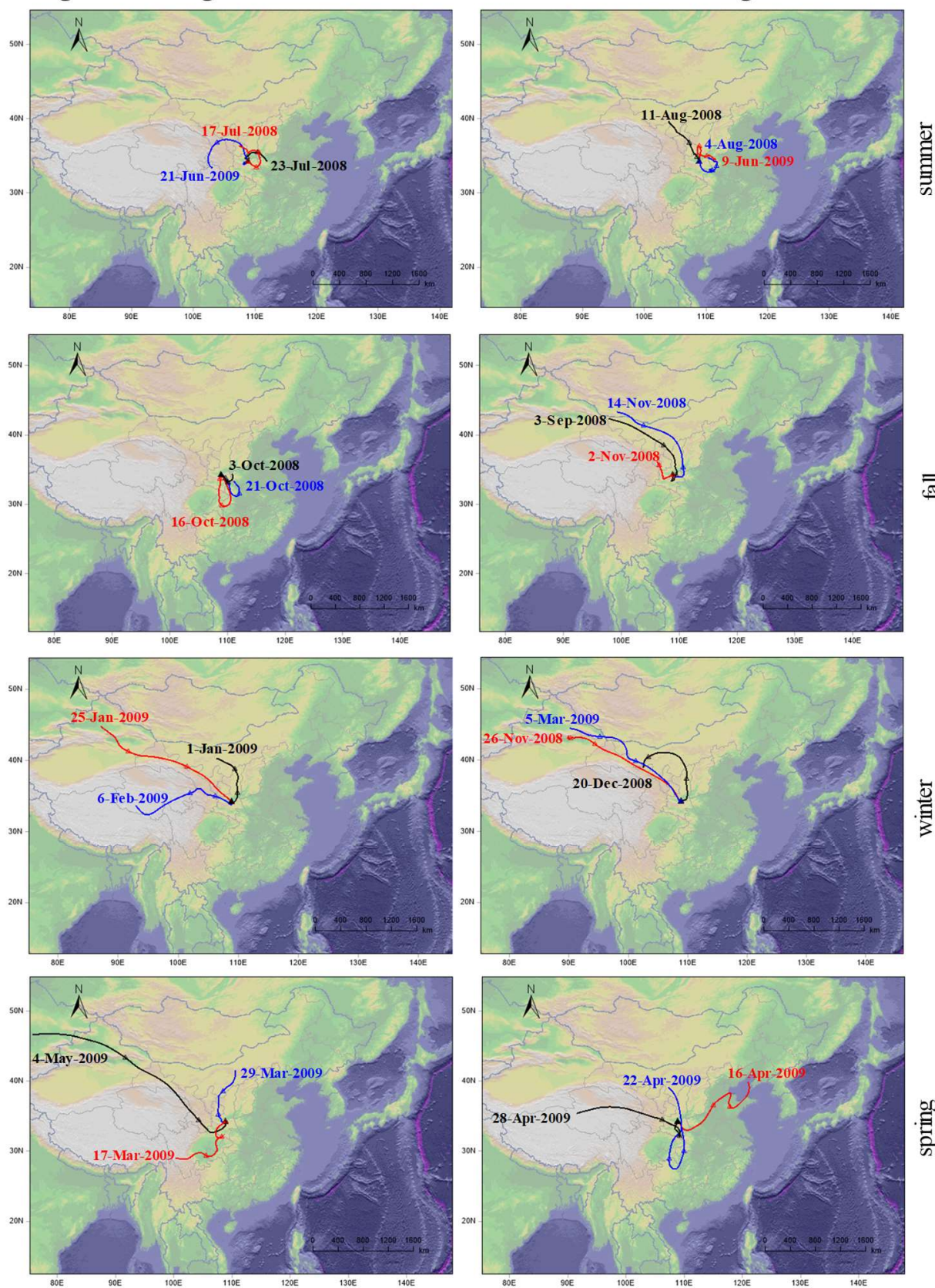

Figure S2. Three-day air backward-in-time air mass trajectory analysis of selected samples for radiocarbon measurements. 


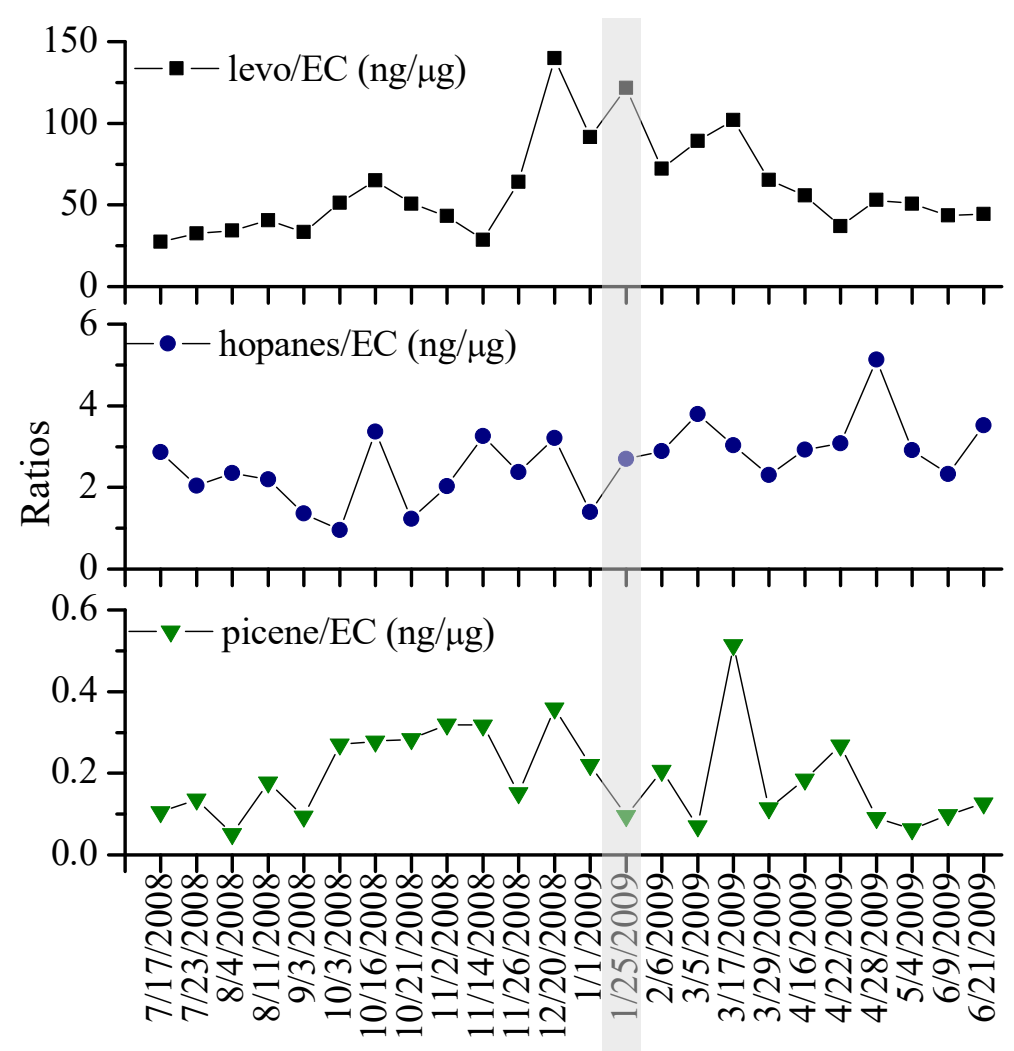

Figure S3. Temporal variation of levoglucosan to EC mass ratios (levo/EC), Shopanes to EC ratios ( hopanes/EC), picene to EC ratios (picene/EC) for samples selected for radiocarbon measurements. Details of measurements are in Supplemental S2. 


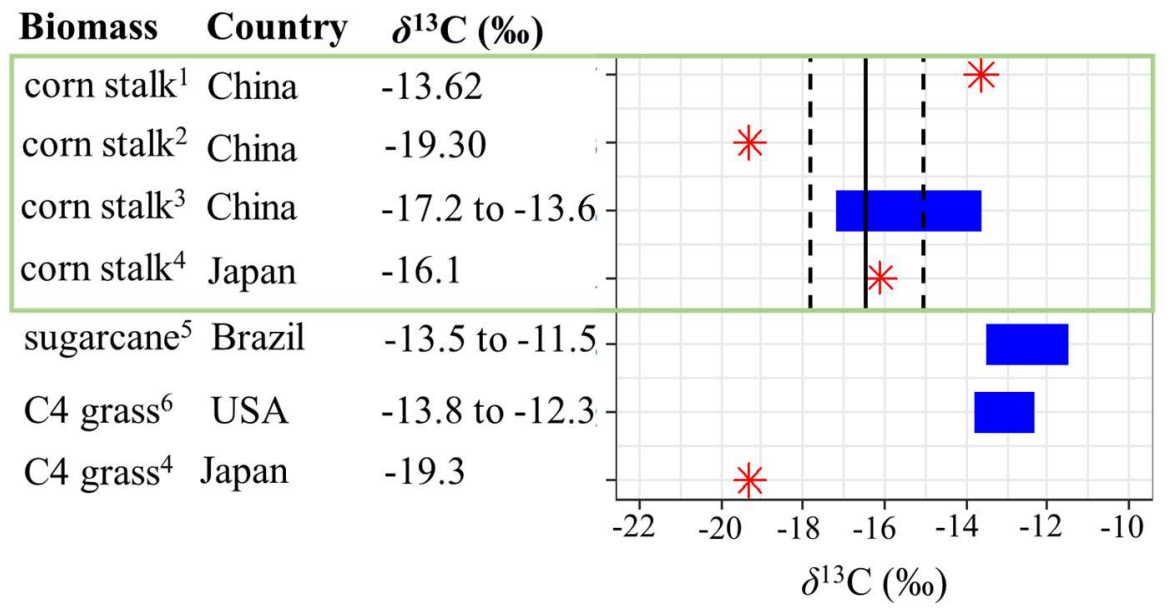

Figure S4. The $\delta^{13} \mathrm{C}$ variability for $\mathrm{C} 4$ plants endmembers. In this study, $\delta^{13} \mathrm{C}$ for corn stalk is used as it is the dominant $\mathrm{C} 4$ plant in $\mathrm{Xi}$ ' an and its surrounding areas (Guanzhong Plain). The range used as $\delta^{13} \mathrm{C}$ of burning corn stalk is indicated as dashed vertical lines, and the mean is shown by a solid vertical line. Sources: ${ }^{1}$ Chen et al. (2012), ${ }^{2}$ Guo et al. (2016), ${ }^{3}$ Liu et al. (2014), ${ }^{4}$ Kawashima and Haneishi (2012), ${ }^{5}$ Martinelli et al. (2002), ${ }^{6}$ Das et al. (2010). 

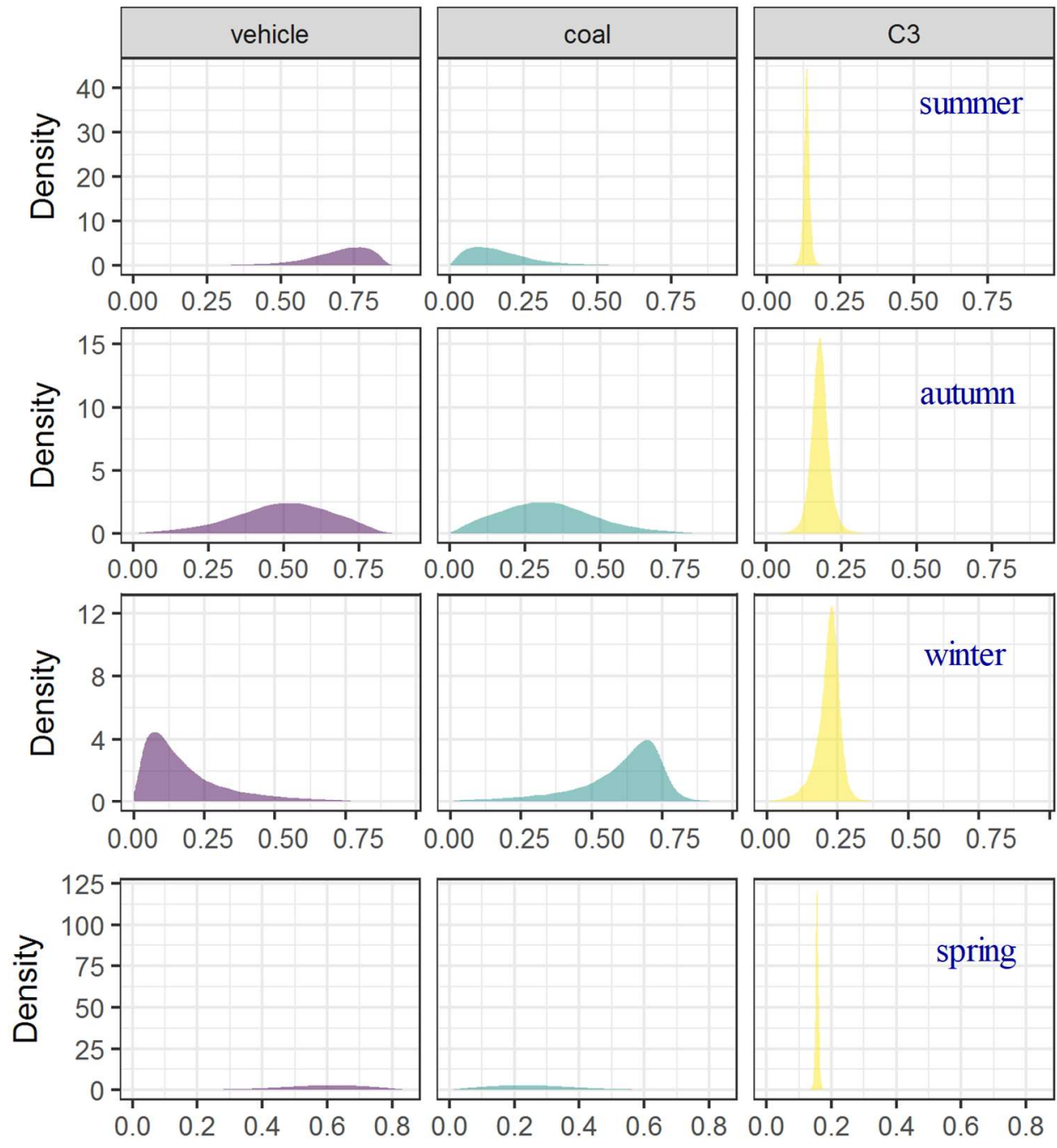

Proportion

Figure S5. MCMC3-derived posterior probability density functions (PDF) of the relative source contributions of C3 plants (denoted as C3), coal and liquid fossil fuel combustion (vehicle) to EC in different seasons, calculated using the Bayesian Markov chain Monte Carlo approach. 
(a)

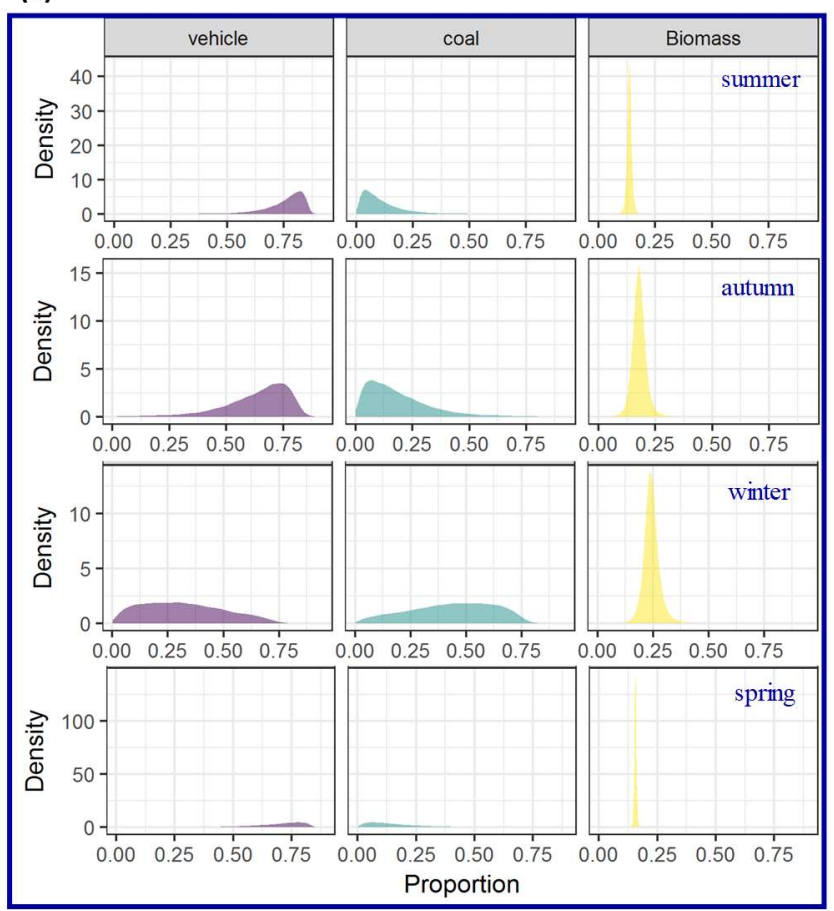

(b)

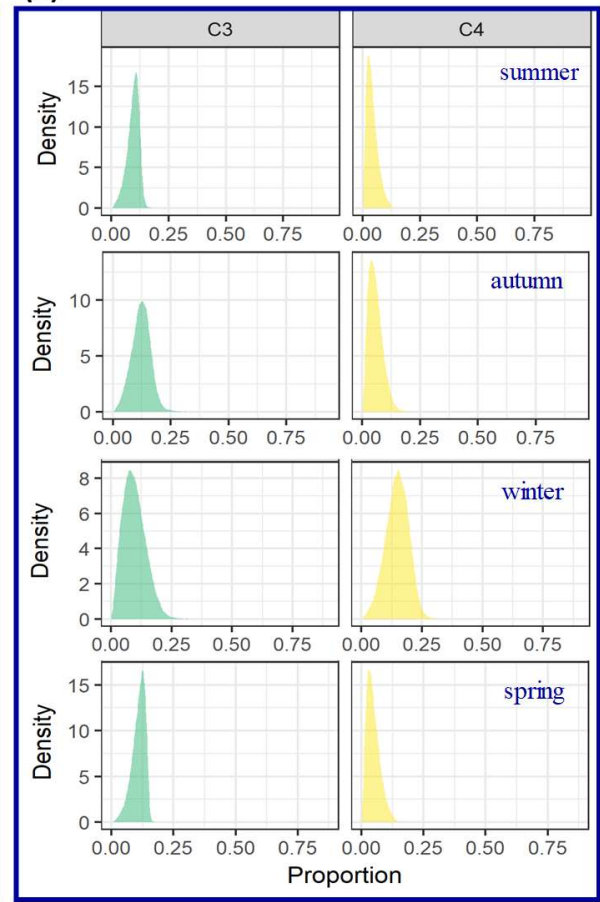

Figure S6. MCMC4-derived posterior probability density functions (PDF) of the relative source contributions of liquid fossil fuel combustion (vehicle), coal and biomass burning (C3 and $\mathrm{C} 4$ plants, denoted as biomass) to EC in different seasons (a), calculated using the Bayesian Markov chain Monte Carlo approach. The PDF of the relative source contributions of biomass burning (a) is a posteriori combination of PDF for C3 plants and C4 plants, as shown in panel (b). 


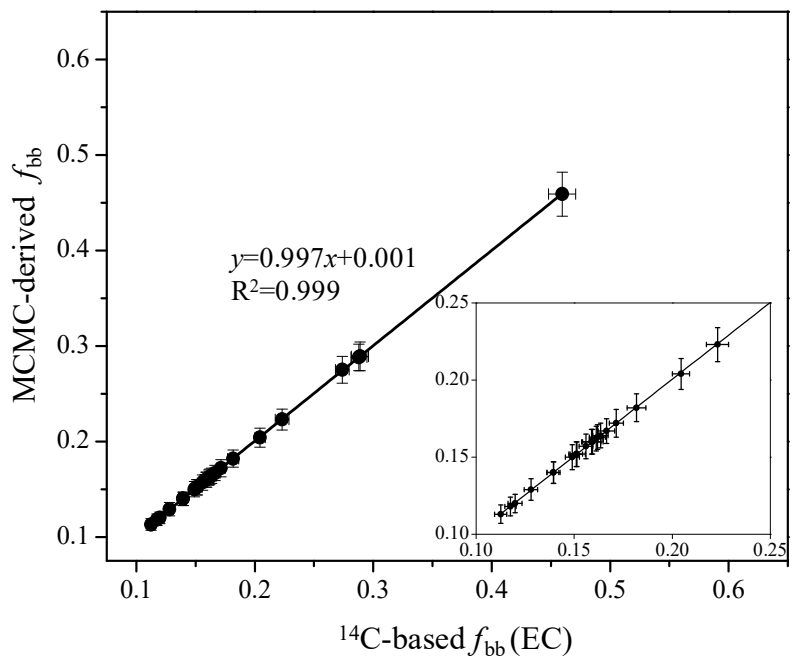

Figure S7. Comparison between the MCMC-derived fraction of biomass burning EC (MCMC-derived $f_{\mathrm{bb}}$ ) and that obtained from radiocarbon data $\left({ }^{14} \mathrm{C}\right.$-based $\left.f_{\mathrm{bb}}(\mathrm{EC})\right)$. Error bars represent one standard deviation. 


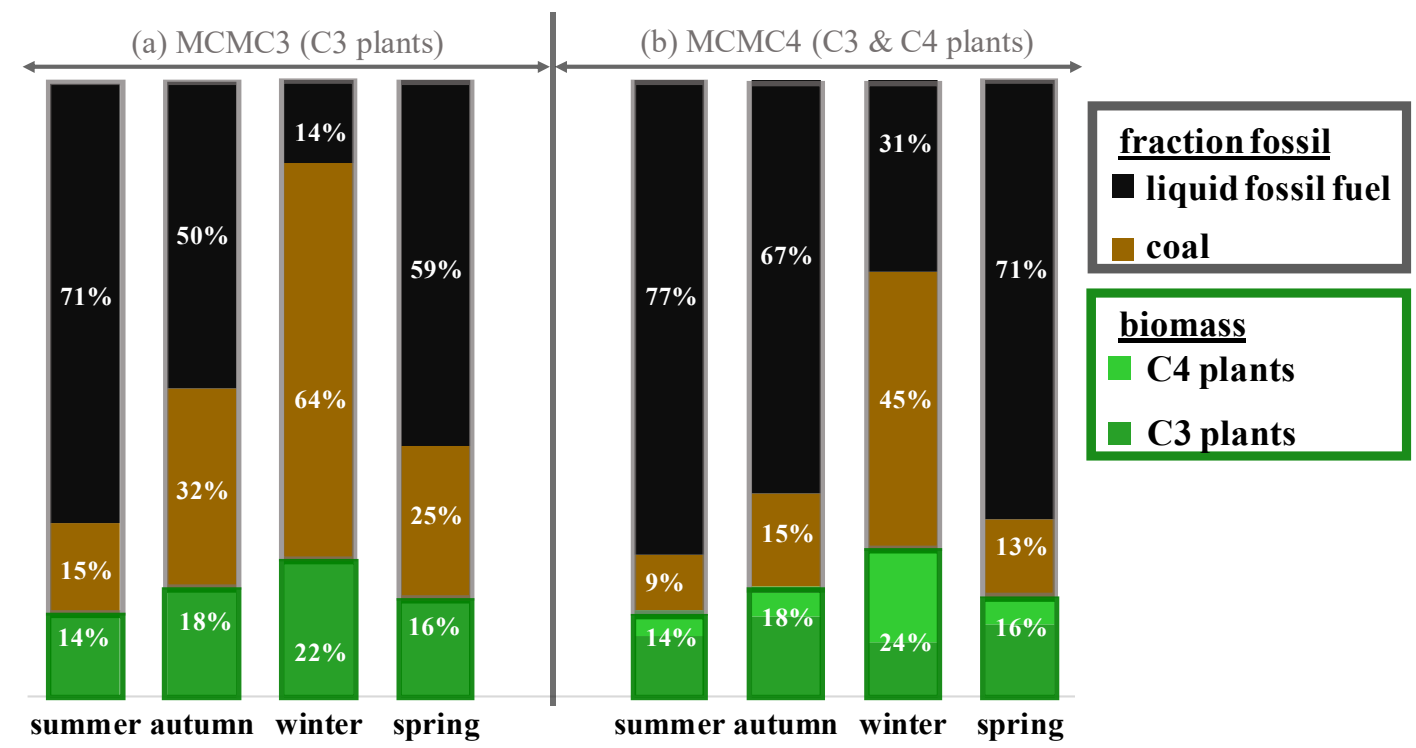

Figure S8. Sources of $\mathrm{EC}$ in different seasons. Results from the $\mathrm{F}^{14} \mathrm{C}$ and $\delta^{13} \mathrm{C}$ based Bayesian source apportionment calculations of EC. The numbers in the bars represent the median contribution of liquid fossil fuel, coal and biomass burning. (a) results from the MCMC3 model, including $\mathrm{C} 3$ plants as biomass, coal and liquid fossil fuel; (b) Impact of C4 plants burning on EC source apportionment is tested by including C4 biomass into the calculations (MCMC4). For MCMC4, the PDF for $\mathrm{C} 3$ and $\mathrm{C} 4$ plants is combined and named as biomass burning. Bars filled with green colour indicate the relative contribution of biomass burning, including C3 plants (light green) and C4 plants (dark green). In winter, the sample taken on Chinese New Year eve (25 January 2009) was excluded. 

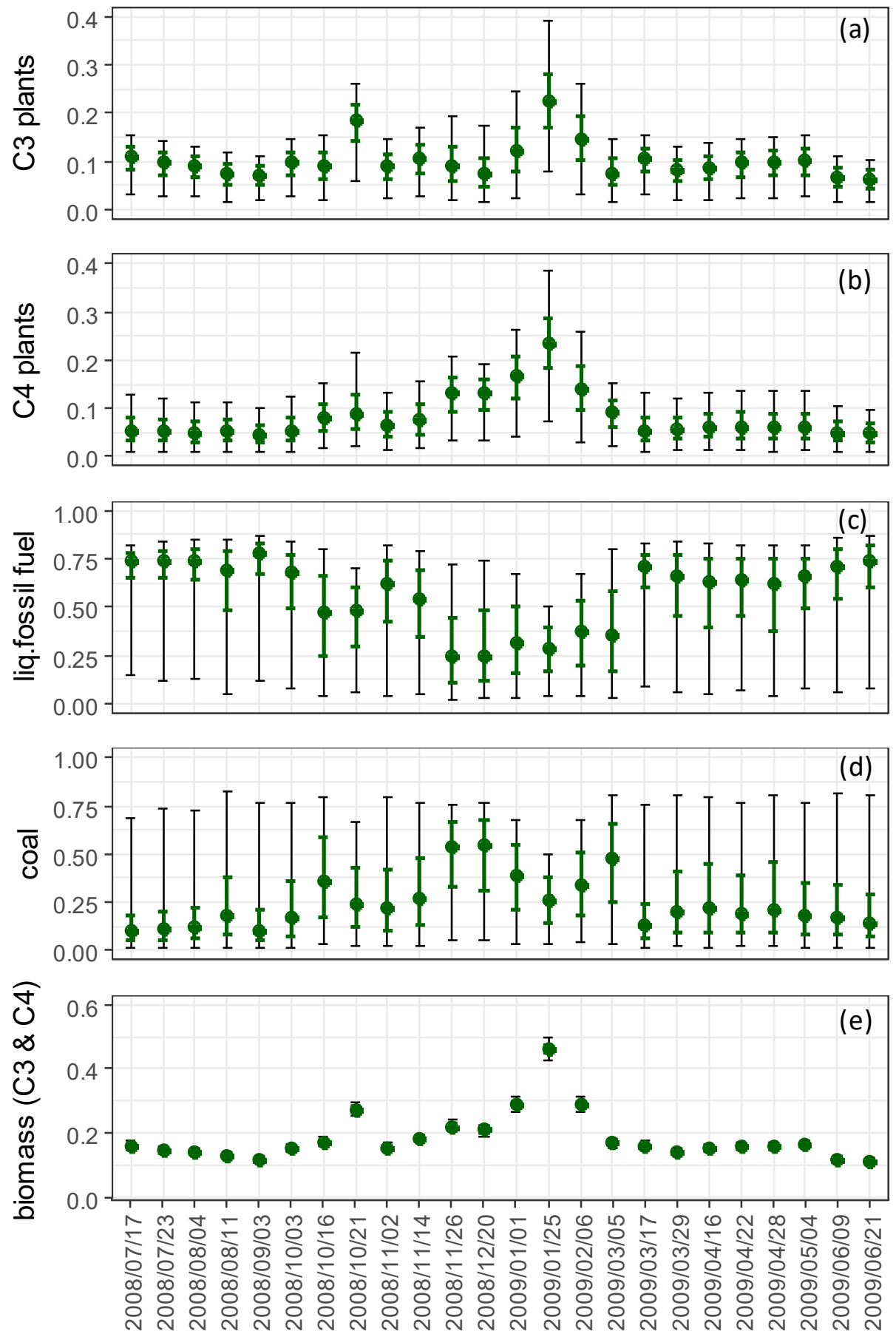

Figure S9. MCMC4-derived source contributions to EC for each data point computed using the Bayesian Markov chain Monte Carlo approach. (a). biomass burning from C3 plants; (b). biomass burning from C4 plants; (c). liquid fossil fuel combustion; (d). coal combustion. Range of $95 \%$ credible intervals (Bayesian analogue of confidence intervals) and interquartile range $\left(25^{\text {th }}-75^{\text {th }}\right.$ percentile) from the computed probability density functions (PDF) and shown in black and green error bars, respectively. To better compare results with MCMC3, we did a posteriori combination of PDF for $\mathrm{C} 3$ biomass (a) and C4 biomass (b) and named the combined PDF as biomass burning (e). 


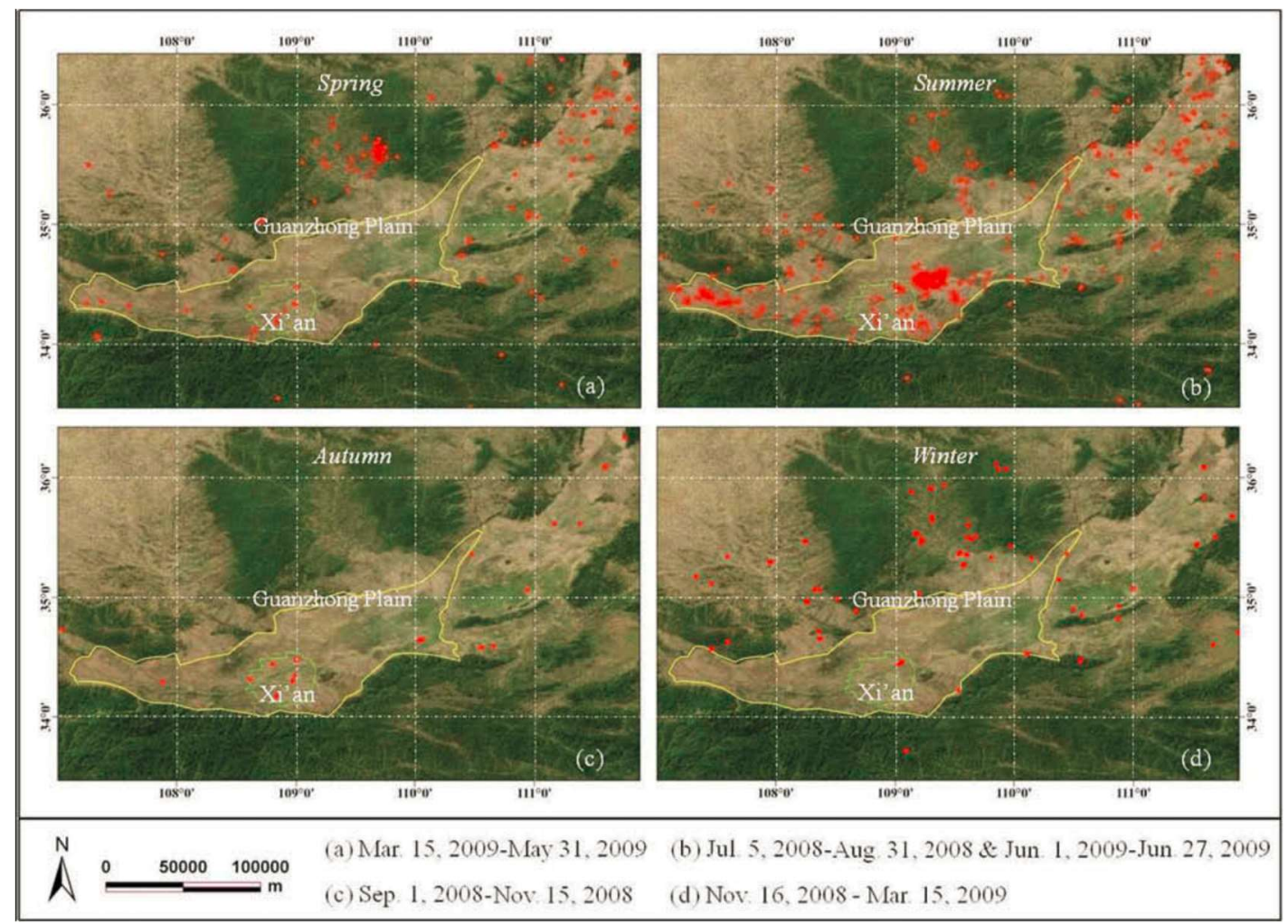

Figure S10. Fire counts (red points) monitored by MODIS in different seasons during the sampling period (https://firms.modaps.eosdis.nasa.gov/firemap/). The sampling site is Xi'an. Xi'an is located in the Guanzhong Plain, one of the major agricultural production areas. 

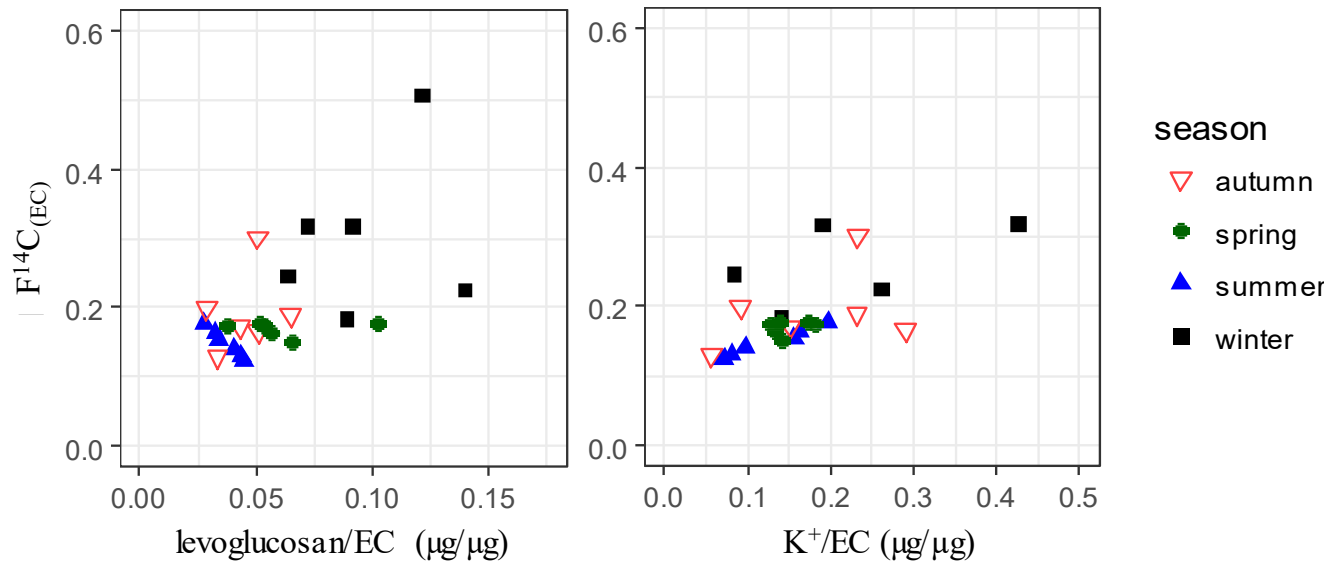

Figure S11. Correlation between $\mathrm{F}^{14} \mathrm{C}_{(\mathrm{EC})}$ and levoglucosan/EC ratios, $\mathrm{K}^{+} / \mathrm{EC}$ ratios in different seasons (red: autumn; dark green: spring; blue: summer; winter: black). One data point with extremely high $\mathrm{K}^{+}$concentration on Chinese New Year is removed. 


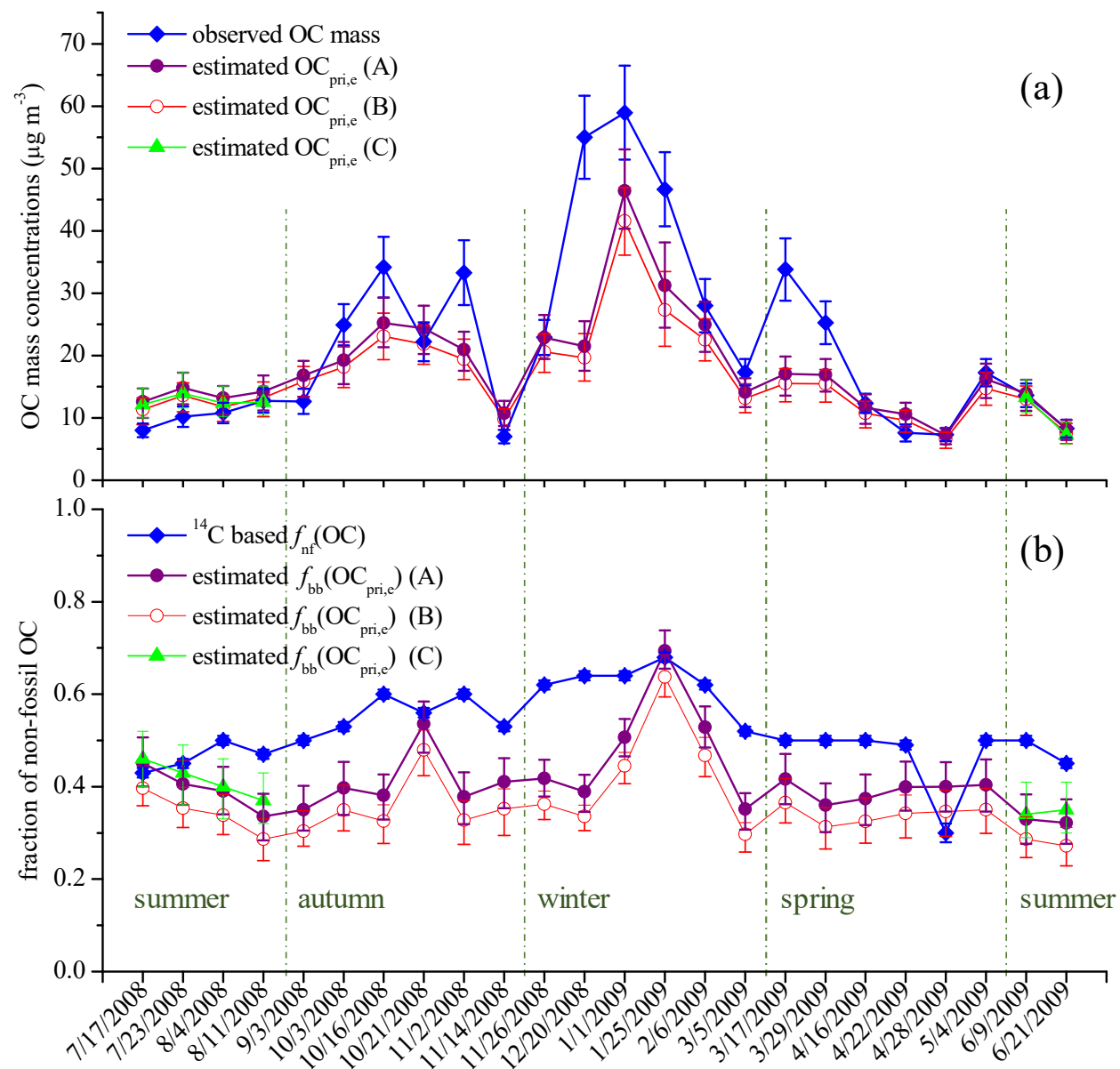

Figure S12. Estimated primary OC based on MCMC3 results. (a) measured OC concentrations (blue line and diamond symbols) with observational uncertainties (vertical bar) and estimated $\mathrm{OC}$ mass (OC triangular symbols) from apportioned EC and OC/EC ratios for different sources (Eq. (10)). (b) ${ }^{14} \mathrm{C}$-based fraction of non-fossil OC $\left(f_{\text {nf }}(\mathrm{OC})\right)$ and modelled non-fossil fraction in $\mathrm{OC}_{\text {pri,e }}\left(f_{\mathrm{bb}}\left(\mathrm{OC}_{\mathrm{pri}, \mathrm{e}}\right)\right)$ derived from Eq. (11). Interquartile range $\left(25^{\text {th }}-75^{\text {th }}\right.$ percentile $)$ of the median $\mathrm{OC}_{\text {priee }}$ and $f_{\mathrm{bb}}\left(\mathrm{OC}_{\text {pri,e }}\right)$ are shown in purple $(\mathrm{A})$, red $(\mathrm{B})$ and green $(\mathrm{C})$ vertical bars. "A" and "B" denotes different $\mathrm{OC} / \mathrm{EC}$ ratios applied to primary biomass burning emissions $\left(r_{\mathrm{bb}}\right)$ : A. $r_{\mathrm{bb}}=5\left(3-7\right.$, minimum-maximum), B. $r_{\mathrm{bb}}=4(3-5)$. "C" denotes $80 \% r_{\text {liq.fossil }}$ applied in summer with $r_{\mathrm{bb}}=$ 5. $f_{\mathrm{nf}}(\mathrm{OC})$ uncertainties are shown but not visible. 


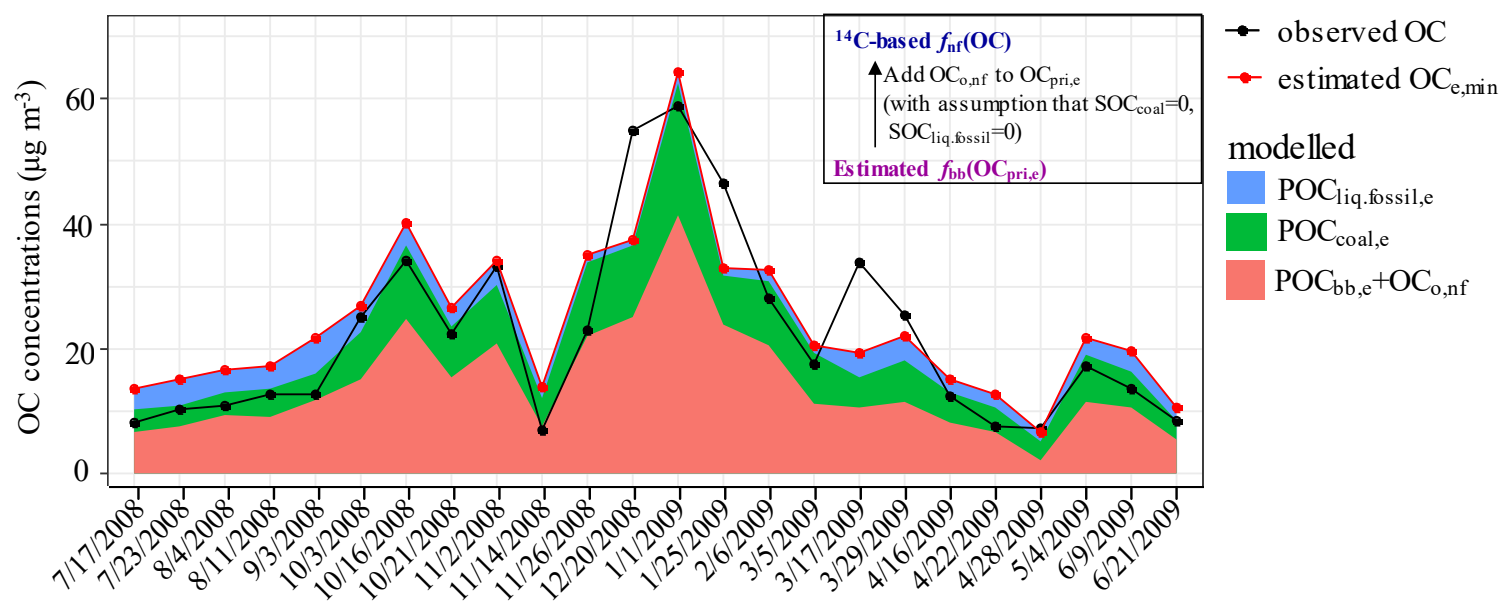

Figure S13. Observed and estimated $\mathrm{OC}$ concentrations. Modelled $\mathrm{OC}_{\mathrm{e}, \mathrm{min}}$ is the sum of $\mathrm{OC}_{\mathrm{pri}, \mathrm{e}}$ and $\mathrm{OC}_{\mathrm{o}, \mathrm{nf}}$. $\mathrm{OC}_{\mathrm{o}, \mathrm{nf}}$ accounts for the differences between $f_{\mathrm{nf}}(\mathrm{OC})$ and $f_{\mathrm{bb}}\left(\mathrm{OC}_{\mathrm{pri}, e}\right)$, with an unrealistic assumption of no secondary fossil $\mathrm{OC}$, leading to minimum addition to $\mathrm{OC}_{\text {pri,e. }}$ Coral area shows the $\mathrm{POC}_{\mathrm{bb}, \mathrm{e}}$ and $\mathrm{OC}_{\mathrm{o}, \mathrm{nf}}$, green area the $\mathrm{POC}_{\mathrm{coal}}$, and

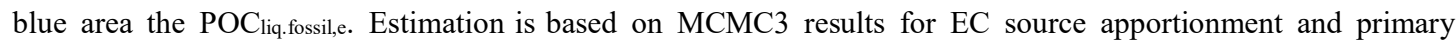
OC/EC ratios corresponding to case (A) in Fig. S12. 


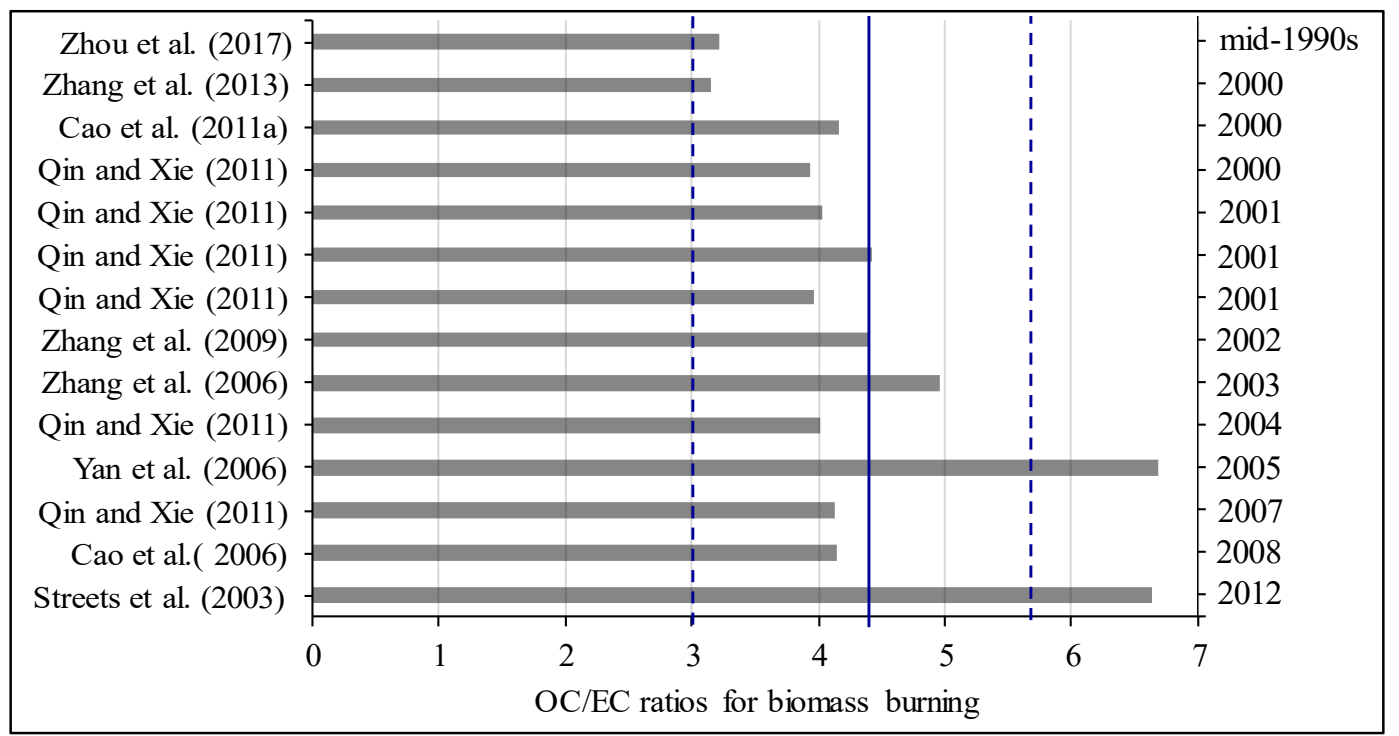

Figure S14. OC/EC ratios estimated from $\mathrm{OC}$ and $\mathrm{EC}$ emission amounts from biomass burning emission inventories specific to China. y-axis on the right side indicates the year of estimation. The range applied in OC estimation (Sect. 3.5 in main text) is shown by dashed vertical lines, and the mean is indicated by a full vertical line. Data sources: Streets et al. (2003); Yan et al. (2006); Zhang et al. (2006, 2009); Cao et al. (2006, 2011a), Qin and Xie (2011), Zhang et al. (2013), Zhou et al. (2017). 


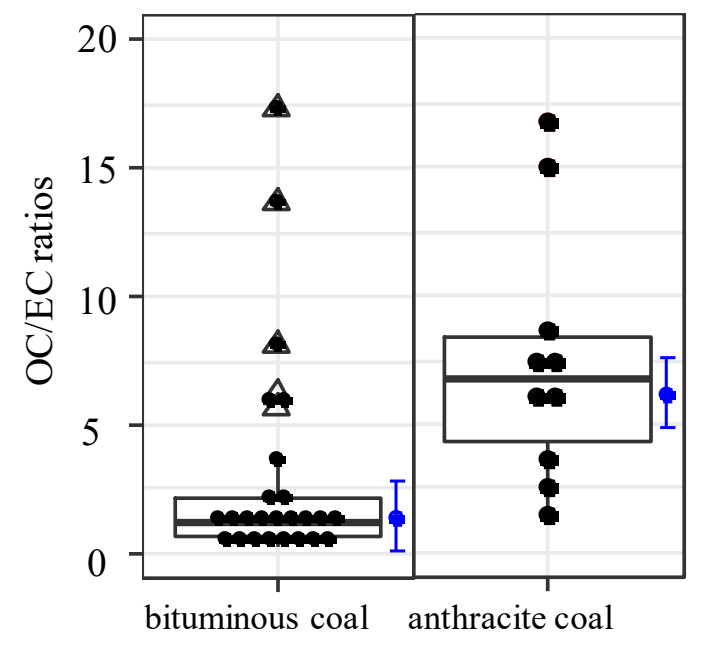

Figure S15. Literature reported OC/EC ratios for combustion of bituminous coal and anthracite coal. Boxplots show the median (thick line across the box), interquartile range $\left(25^{\text {th }}-75^{\text {th }}\right.$ percentile, vertical ends of the box). Outliers are shown as triangles. Blue dots (averages) with error bars (one standard deviation) represents OC/EC ratios measured by IMPROVE_A protocol reported by Tian et al. (2017). Data sources: Chen et al. (2005, 2006, 2015), Zhang et al., (2008, 2012), Zhi et al. (2008), Shen et al. (2010, 2015), Li et al. (2016b). 
Table S1. Range of $\delta^{13} \mathrm{C}$ values for each source reported in previous studies

\begin{tabular}{lll}
\hline Sources & $\begin{array}{l}\delta^{13} \mathrm{C}(\%) \text { of } \\
\text { emissions from } \\
\text { sources }\end{array}$ & Reference \\
\hline $\begin{array}{l}\text { C4 plants } \\
\text { (corn, sugar cane, grass) }\end{array}$ & $\begin{array}{l}-19.30 \% \text { to } \\
-11.5 \%{ }^{\mathrm{a}}\end{array}$ & $\begin{array}{l}\text { (Martinelli et al., 2002; Das et al., 2010; Chen et al., } \\
\text { 2012; Kawashima and Haneishi, 2012; Liu et al., } \\
\text { 2014; Guo et al., 2016) }\end{array}$ \\
$\begin{array}{l}\text { C3 plants } \\
\text { (wood, wheat straw, etc.) }\end{array}$ & $-26.7 \pm 1.8 \%$ & Andersson et al. (2015) and references therein \\
$\begin{array}{l}\text { coal } \\
\begin{array}{l}\text { liquid fuel } \\
\text { (e.g., gasoline, diesel, and oil) }\end{array}\end{array}$ & $-23.38 \pm 1.3 \%$ & Andersson et al. (2015) and references therein \\
\hline
\end{tabular}

${ }^{a} \delta^{13} \mathrm{C}$ source signatures for emissions from burning corn stalk (C4 plant) of $-16.45 \pm 1.4 \%$ (mean \pm standard deviation) are applied in MCMC4 calculations. See details on selection of $\delta^{13} \mathrm{C}$ signature for $\mathrm{C} 4$ plants in the study area (corn stalk) in Sect.3.4.1. 
Table S2. Mass concentrations of PM2.5, OC and EC in Xi' an, China from July 2008 to June 2009.

\begin{tabular}{llll}
\hline & $\mathrm{PM}_{2.5}\left(\mu \mathrm{g} \mathrm{m}^{-3}\right)$ & $\mathrm{OC}\left(\mu \mathrm{g} \mathrm{m}^{-3}\right)$ & $\mathrm{EC}\left(\mu \mathrm{g} \mathrm{m}^{-3}\right)$ \\
\hline Spring $(\mathrm{n}=12)$ & $124.0 \pm 40.4(55.9-193.4)^{a}$ & $14.4 \pm 9.6(3.3-33.8)$ & $5.7 \pm 2.3(2.0-8.8)$ \\
Summer $(\mathrm{n}=22)$ & $83.0 \pm 30.7(31.8-139.2)$ & $12.7 \pm 4.5(4.0-20.6)$ & $6.3 \pm 2.0(2.7-10.0)$ \\
Autumn $(\mathrm{n}=12)$ & $125.1 \pm 69.3(41.0-212.6)$ & $22.2 \pm 13.6(3.6-34.2)$ & $8.4 \pm 2.9(3.5-11.3)$ \\
Winter $(\mathrm{n}=19)$ & $213.4 \pm 91.8(73.1-408.5)$ & $39.0 \pm 17.8(10.8-67.0)$ & $9.1 \pm 3.1(5.6-16.3)$ \\
\hline Annual & $142.0 \pm 82.4(31.8-408.5)$ & $21.5 \pm 16.6(3.3-67.0)$ & $7.6 \pm 3.0(2.0-16.3)$ \\
\hline
\end{tabular}

${ }^{a}$ average \pm standard deviation, the number in the parentheses is the range of each dataset. 
Table S3. Average fraction modern $\left(\mathrm{F}^{14} \mathrm{C}\right)$ and stable carbon signature $\left(\delta^{13} \mathrm{C}\right)$ of $\mathrm{OC}$ and $\mathrm{EC}$ for selected samples.

\begin{tabular}{|c|c|c|c|c|c|}
\hline Date & $\mathrm{F}^{14} \mathrm{C}_{(\mathrm{OC})}$ & $\mathrm{F}^{14} \mathrm{C}_{(\mathrm{EC})}$ & $\delta^{13} \mathrm{CoC}$ & $\delta^{13} \mathrm{C}_{\mathrm{EC}}$ & Season \\
\hline $7 / 17 / 2008^{a}$ & $0.466 \pm 0.010$ & $0.178 \pm 0.003$ & -26.80 & -26.50 & summer \\
\hline $7 / 23 / 2008$ & $0.489 \pm 0.008$ & $0.164 \pm 0.003$ & -25.94 & -26.33 & summer \\
\hline $8 / 4 / 2008$ & $0.546 \pm 0.007$ & $0.153 \pm 0.002$ & -25.86 & -26.16 & summer \\
\hline $8 / 11 / 2008$ & $0.512 \pm 0.008$ & $0.141 \pm 0.003$ & -25.21 & -25.53 & summer \\
\hline $9 / 3 / 2008$ & $0.549 \pm 0.006$ & $0.129 \pm 0.002$ & -25.94 & -26.23 & autumn \\
\hline $10 / 3 / 2008$ & $0.581 \pm 0.006$ & $0.166 \pm 0.002$ & -24.55 & -25.51 & autumn \\
\hline $10 / 16 / 2008$ & $0.659 \pm 0.007$ & $0.188 \pm 0.002$ & -23.70 & -24.31 & autumn \\
\hline $10 / 21 / 2008$ & $0.610 \pm 0.005$ & $0.301 \pm 0.003$ & -24.51 & -24.92 & autumn \\
\hline $11 / 2 / 2008$ & $0.651 \pm 0.006$ & $0.172 \pm 0.002$ & -24.94 & -25.10 & autumn \\
\hline $11 / 14 / 2008$ & $0.579 \pm 0.007$ & $0.200 \pm 0.004$ & -25.48 & -24.79 & autumn \\
\hline $11 / 26 / 2008$ & $0.671 \pm 0.009$ & $0.245 \pm 0.004$ & -24.71 & -22.93 & winter \\
\hline $12 / 20 / 2008$ & $0.696 \pm 0.008$ & $0.225 \pm 0.002$ & -24.06 & -22.81 & winter \\
\hline $1 / 1 / 2009$ & $0.693 \pm 0.007$ & $0.317 \pm 0.004$ & -23.23 & -23.12 & winter \\
\hline $1 / 25 / 2009$ & $0.745 \pm 0.005$ & $0.505 \pm 0.008$ & -23.39 & -23.07 & winter \\
\hline 2/6/2009 & $0.671 \pm 0.007$ & $0.318 \pm 0.005$ & -23.92 & -23.72 & winter \\
\hline $3 / 5 / 2009$ & $0.572 \pm 0.006$ & $0.183 \pm 0.003$ & -25.44 & -23.53 & winter \\
\hline $3 / 17 / 2009$ & $0.545 \pm 0.004$ & $0.177 \pm 0.002$ & -25.72 & -26.03 & spring \\
\hline $3 / 29 / 2009$ & $0.547 \pm 0.006$ & $0.153 \pm 0.002$ & -26.91 & -25.38 & spring \\
\hline $4 / 16 / 2009$ & $0.545 \pm 0.007$ & $0.166 \pm 0.003$ & -27.42 & -25.05 & spring \\
\hline $4 / 22 / 2009$ & $0.535 \pm 0.006$ & $0.175 \pm 0.004$ & -26.33 & -25.27 & spring \\
\hline $4 / 28 / 2009$ & $0.330 \pm 0.021$ & $0.175 \pm 0.005$ & -26.41 & -25.33 & spring \\
\hline $5 / 4 / 2009$ & $0.544 \pm 0.004$ & $0.180 \pm 0.003$ & -26.66 & -25.35 & spring \\
\hline 6/9/2009 & $0.549 \pm 0.006$ & $0.132 \pm 0.003$ & -24.24 & -25.37 & summer \\
\hline $6 / 21 / 2009$ & $0.489 \pm 0.006$ & $0.124 \pm 0.002$ & -26.30 & -25.73 & summer \\
\hline summer $^{\mathrm{b}}$ & $0.509 \pm 0.033$ & $0.149 \pm 0.020$ & $-25.73 \pm 0.90$ & $-25.94 \pm 0.46$ & \\
\hline autumn & $0.605 \pm 0.044$ & $0.193 \pm 0.058$ & $-24.85 \pm 0.79$ & $-25.14 \pm 0.66$ & \\
\hline winter & $0.675 \pm 0.057$ & $0.299 \pm 0.114$ & $-24.13 \pm 0.83$ & $-23.20 \pm 0.35$ & \\
\hline spring & $0.508 \pm 0.087$ & $0.171 \pm 0.010$ & $-26.58 \pm 0.57$ & $-25.40 \pm 0.33$ & \\
\hline
\end{tabular}

${ }^{a}$ Daily $\mathrm{F}^{14} \mathrm{C}$ values are given in average \pm measurement uncertainties;

${ }^{\mathrm{b}}$ Seasonal averaged $\mathrm{F}^{14} \mathrm{C}$ and $\delta^{13} \mathrm{C}$ values are given in average \pm standard deviation. 
Table S4. Average $\mathrm{OC}$ and $\mathrm{EC}$ concentrations from non-fossil sources $\left(\mathrm{OC}_{\mathrm{nf}}, \mathrm{EC} \mathrm{bb}\right)$ and fossil sources $\left(\mathrm{OC}_{\text {fossil, }}\right.$ EC fossil), relative non-fossil sources contribution to $\mathrm{OC}$ and $\mathrm{EC}\left(f_{\mathrm{nf}}(\mathrm{OC}), f_{\mathrm{bb}}(\mathrm{EC})\right)$, and relative fossil sources contribution to $\mathrm{OC}$ and $\mathrm{EC}\left(f_{\text {fossil }}(\mathrm{OC}), f_{\text {fossil }}(\mathrm{EC})\right)$.

\begin{tabular}{|c|c|c|c|c|c|c|c|c|c|}
\hline Date & $\mathrm{OC}_{\mathrm{nf}}$ & $\mathrm{OC}_{\text {fossil }}$ & $\mathrm{EC}_{\mathrm{bb}}$ & $\mathrm{EC}_{\text {fossil }}$ & $f_{\mathrm{nf}}(\mathrm{OC})$ & $f_{\text {fossil }}(\mathrm{OC})$ & $f_{\mathrm{bb}}(\mathrm{EC})$ & $f_{\text {fossil }}(\mathrm{EC})$ & Season \\
\hline $7 / 17 / 2008$ & $3.53 \pm 0.50$ & $4.48 \pm 0.63$ & $1.13 \pm 0.26$ & $5.87 \pm 1.29$ & $0.440 \pm 0.010$ & $0.560 \pm 0.010$ & $0.162 \pm 0.004$ & $0.838 \pm 0.004$ & summer \\
\hline $7 / 23 / 2008$ & $4.70 \pm 0.75$ & $5.49 \pm 0.89$ & $1.20 \pm 0.24$ & $6.84 \pm 1.41$ & $0.461 \pm 0.009$ & $0.539 \pm 0.009$ & $0.149 \pm 0.004$ & $0.851 \pm 0.004$ & summer \\
\hline $8 / 4 / 2008$ & $5.56 \pm 0.85$ & $5.22 \pm 0.80$ & $1.01 \pm 0.21$ & $6.21 \pm 1.26$ & $0.515 \pm 0.008$ & $0.485 \pm 0.008$ & $0.139 \pm 0.003$ & $0.861 \pm 0.003$ & summer \\
\hline $8 / 11 / 2008$ & $6.16 \pm 0.93$ & $6.59 \pm 0.99$ & $0.96 \pm 0.21$ & $6.51 \pm 1.41$ & $0.483 \pm 0.009$ & $0.517 \pm 0.009$ & $0.128 \pm 0.003$ & $0.872 \pm 0.003$ & summer \\
\hline $9 / 3 / 2008$ & $6.54 \pm 1.05$ & $6.09 \pm 0.98$ & $1.17 \pm 0.18$ & $8.81 \pm 1.32$ & $0.518 \pm 0.007$ & $0.482 \pm 0.007$ & $0.117 \pm 0.003$ & $0.883 \pm 0.003$ & autumn \\
\hline $10 / 3 / 2008$ & $13.65 \pm 1.80$ & $11.26 \pm 1.50$ & $1.54 \pm 0.31$ & $8.64 \pm 1.75$ & $0.548 \pm 0.007$ & $0.452 \pm 0.007$ & $0.151 \pm 0.003$ & $0.849 \pm 0.003$ & autumn \\
\hline $10 / 16 / 2008$ & $21.23 \pm 3.11$ & $12.95 \pm 1.86$ & $1.94 \pm 0.32$ & $9.40 \pm 1.57$ & $0.622 \pm 0.008$ & $0.378 \pm 0.008$ & $0.171 \pm 0.004$ & $0.829 \pm 0.004$ & autumn \\
\hline $10 / 21 / 2008$ & $12.82 \pm 1.78$ & $9.41 \pm 1.32$ & $2.65 \pm 0.44$ & $7.03 \pm 1.13$ & $0.576 \pm 0.007$ & $0.424 \pm 0.007$ & $0.274 \pm 0.006$ & $0.726 \pm 0.006$ & autumn \\
\hline $11 / 2 / 2008$ & $20.42 \pm 3.22$ & $12.82 \pm 2.03$ & $1.60 \pm 0.22$ & $8.62 \pm 1.20$ & $0.614 \pm 0.008$ & $0.386 \pm 0.008$ & $0.156 \pm 0.003$ & $0.844 \pm 0.003$ & autumn \\
\hline $11 / 14 / 2008$ & $3.83 \pm 0.60$ & $3.16 \pm 0.50$ & $0.89 \pm 0.18$ & $4.00 \pm 0.78$ & $0.546 \pm 0.008$ & $0.454 \pm 0.008$ & $0.182 \pm 0.005$ & $0.818 \pm 0.005$ & autumn \\
\hline $11 / 26 / 2008$ & $14.49 \pm 1.80$ & $8.41 \pm 1.05$ & $1.91 \pm 0.36$ & $6.66 \pm 1.25$ & $0.634 \pm 0.010$ & $0.366 \pm 0.010$ & $0.223 \pm 0.006$ & $0.777 \pm 0.006$ & winter \\
\hline $12 / 20 / 2008$ & $36.16 \pm 4.43$ & $18.83 \pm 2.37$ & $1.69 \pm 0.43$ & $6.56 \pm 1.69$ & $0.657 \pm 0.009$ & $0.343 \pm 0.009$ & $0.204 \pm 0.004$ & $0.796 \pm 0.004$ & winter \\
\hline $1 / 1 / 2009$ & $38.59 \pm 4.92$ & $20.39 \pm 2.65$ & $4.69 \pm 0.71$ & $11.62 \pm 1.72$ & $0.654 \pm 0.008$ & $0.346 \pm 0.008$ & $0.288 \pm 0.007$ & $0.712 \pm 0.007$ & winter \\
\hline $1 / 25 / 2009$ & $32.79 \pm 4.19$ & $13.86 \pm 1.78$ & $4.34 \pm 1.34$ & $5.10 \pm 1.58$ & $0.703 \pm 0.007$ & $0.297 \pm 0.007$ & $0.459 \pm 0.011$ & $0.541 \pm 0.011$ & winter \\
\hline $2 / 6 / 2009$ & $17.71 \pm 2.74$ & $10.27 \pm 1.61$ & $2.68 \pm 0.50$ & $6.58 \pm 1.19$ & $0.633 \pm 0.008$ & $0.367 \pm 0.008$ & $0.289 \pm 0.007$ & $0.711 \pm 0.007$ & winter \\
\hline $3 / 5 / 2009$ & $9.36 \pm 1.15$ & $7.98 \pm 0.98$ & $0.99 \pm 0.22$ & $4.97 \pm 1.08$ & $0.540 \pm 0.007$ & $0.460 \pm 0.007$ & $0.166 \pm 0.004$ & $0.834 \pm 0.004$ & winter \\
\hline $3 / 17 / 2009$ & $17.38 \pm 2.58$ & $16.39 \pm 2.47$ & $1.41 \pm 0.27$ & $7.33 \pm 1.37$ & $0.514 \pm 0.006$ & $0.486 \pm 0.006$ & $0.161 \pm 0.003$ & $0.839 \pm 0.003$ & spring \\
\hline $3 / 29 / 2009$ & $13.05 \pm 1.77$ & $12.21 \pm 1.67$ & $1.22 \pm 0.19$ & $7.56 \pm 1.15$ & $0.517 \pm 0.007$ & $0.483 \pm 0.007$ & $0.139 \pm 0.003$ & $0.861 \pm 0.003$ & spring \\
\hline $4 / 16 / 2009$ & $6.33 \pm 0.80$ & $5.98 \pm 0.76$ & $0.87 \pm 0.19$ & $4.87 \pm 1.07$ & $0.515 \pm 0.008$ & $0.485 \pm 0.008$ & $0.151 \pm 0.004$ & $0.849 \pm 0.004$ & spring \\
\hline $4 / 22 / 2009$ & $3.84 \pm 0.68$ & $3.77 \pm 0.68$ & $0.84 \pm 0.17$ & $4.43 \pm 0.89$ & $0.505 \pm 0.007$ & $0.495 \pm 0.007$ & $0.159 \pm 0.005$ & $0.841 \pm 0.005$ & spring \\
\hline $4 / 28 / 2009$ & $2.28 \pm 0.35$ & $5.03 \pm 0.72$ & $0.58 \pm 0.12$ & $3.08 \pm 0.63$ & $0.311 \pm 0.019$ & $0.689 \pm 0.019$ & $0.159 \pm 0.005$ & $0.841 \pm 0.005$ & spring \\
\hline $5 / 4 / 2009$ & $8.86 \pm 1.12$ & $8.41 \pm 1.08$ & $1.30 \pm 0.25$ & $6.65 \pm 1.30$ & $0.513 \pm 0.006$ & $0.487 \pm 0.006$ & $0.163 \pm 0.004$ & $0.837 \pm 0.004$ & spring \\
\hline 6/9/2009 & $7.07 \pm 0.98$ & $6.57 \pm 0.92$ & $0.91 \pm 0.17$ & $6.72 \pm 1.23$ & $0.518 \pm 0.007$ & $0.482 \pm 0.007$ & $0.120 \pm 0.004$ & $0.880 \pm 0.004$ & summer \\
\hline $6 / 21 / 2009$ & $3.81 \pm 0.66$ & $4.47 \pm 0.78$ & $0.52 \pm 0.13$ & $4.07 \pm 1.02$ & $0.462 \pm 0.006$ & $0.538 \pm 0.006$ & $0.113 \pm 0.003$ & $0.887 \pm 0.003$ & summer \\
\hline
\end{tabular}


Table S5. OC and EC concentrations from non-fossil sources ( $\left.\mathrm{OC}_{\mathrm{nf}}, \mathrm{EC} \mathrm{bb}\right)$ and fossil sources ( $\mathrm{OC}_{\text {fossil, }}$ EC fossil), relative non-fossil sources contribution to $\mathrm{OC}$ and $\mathrm{EC}\left(f_{\mathrm{nf}}(\mathrm{OC})\right.$, $\left.f_{\mathrm{bb}}(\mathrm{EC})\right)$, and relative fossil sources contribution to $\mathrm{OC}$ and $\mathrm{EC}\left(f_{\text {fossil }}(\mathrm{OC}), f_{\text {fossil }}(\mathrm{EC})\right)$ in different seasons.

\begin{tabular}{|c|c|c|c|c|c|c|c|c|}
\hline Season & $\mathrm{OC}_{\mathrm{nf}}$ & $\mathrm{OC}_{\text {fossil }}$ & $\mathrm{EC}_{\mathrm{bb}}$ & $\mathrm{EC}_{\text {fossil }}$ & $f_{\mathrm{nf}}(\mathrm{OC})$ & $f_{\text {fossil }}(\mathrm{OC})$ & $f_{\mathrm{bb}}(\mathrm{EC})$ & $f_{\text {fossil }}(\mathrm{EC})$ \\
\hline \multirow[t]{2}{*}{ summer } & $5.14 \pm 1.38^{\mathrm{a}}$ & $5.47 \pm 0.95$ & $0.95 \pm 0.24$ & $6.03 \pm 1.02$ & $0.480 \pm 0.032$ & $0.520 \pm 0.032$ & $0.135 \pm 0.018$ & $0.865 \pm 0.018$ \\
\hline & $(3.53 \sim 7.07)$ & $(4.47 \sim 6.59)$ & $(0.52 \sim 1.20)$ & $(4.07 \sim 6.84)$ & $(0.440 \sim 0.518)$ & $(0.482 \sim 0.560)$ & $(0.113 \sim 0.162)$ & $(0.838 \sim 0.887)$ \\
\hline \multirow[t]{2}{*}{ autumn } & $13.08 \pm 7.06$ & $9.28 \pm 3.94$ & $1.63 \pm 0.62$ & $7.75 \pm 2.00$ & $0.571 \pm 0.041$ & $0.429 \pm 0.041$ & $0.175 \pm 0.053$ & $0.825 \pm 0.053$ \\
\hline & $(3.83 \sim 21.23)$ & $(3.16 \sim 12.95)$ & $(0.89 \sim 2.65)$ & $(4.00 \sim 9.40)$ & $(0.518 \sim 0.622)$ & $(0.378 \sim 0.482)$ & $(0.117 \sim 0.274)$ & $(0.726 \sim 0.883)$ \\
\hline \multirow[t]{2}{*}{ winter ${ }^{b}$} & $23.26 \pm 13.25$ & $13.18 \pm 5.96$ & $2.39 \pm 1.42$ & $7.28 \pm 2.53$ & $0.624 \pm 0.048$ & $0.376 \pm 0.048$ & $0.234 \pm 0.054$ & $0.766 \pm 0.054$ \\
\hline & $(9.36 \sim 38.59)$ & $(7.98 \sim 20.39)$ & $(0.99 \sim 4.69)$ & $(4.97 \sim 11.62)$ & $(0.540 \sim 0.657)$ & $(0.343 \sim 0.460)$ & $(0.166 \sim 0.289)$ & $(0.711 \sim 0.834)$ \\
\hline \multirow[t]{2}{*}{ spring } & $8.62 \pm 5.74$ & $8.63 \pm 4.83$ & $1.04 \pm 0.32$ & $5.65 \pm 1.80$ & $0.479 \pm 0.082$ & $0.521 \pm 0.082$ & $0.155 \pm 0.009$ & $0.845 \pm 0.009$ \\
\hline & $(2.28 \sim 17.38)$ & $(3.77 \sim 16.39)$ & $(0.58 \sim 1.41)$ & $(3.08 \sim 7.56)$ & $(0.311 \sim 0.517)$ & $(0.483 \sim 0.689)$ & $(0.139 \sim 0.163)$ & $(0.837 \sim 0.861)$ \\
\hline \multirow[t]{2}{*}{ overall $^{\mathrm{b}}$} & $12.06 \pm 9.81$ & $8.96 \pm 4.79$ & $1.46 \pm 0.90$ & $6.65 \pm 1.96$ & $0.535 \pm 0.080$ & $0.465 \pm 0.080$ & $0.172 \pm 0.051$ & $0.828 \pm 0.051$ \\
\hline & $(2.28 \sim 38.59)$ & $(3.16 \sim 20.39)$ & $(0.52 \sim 4.69)$ & $(3.08 \sim 11.62)$ & $(0.311 \sim 0.657)$ & $(0.343 \sim 0.689)$ & $(0.113 \sim 0.289)$ & $(0.711 \sim 0.887)$ \\
\hline
\end{tabular}

${ }^{a}$ data is given in average \pm standard deviation, minimum and maximum are shown in parentheses

$\mathrm{b}$ the sample taken on Chinese New Year eve (25 January 2009) was excluded. 
Table S6. $\mathrm{MCMC} 3$ results ${ }^{\mathrm{a}}$ from the $\mathrm{F}^{14} \mathrm{C}$ - and $\delta^{13} \mathrm{C}$-based Bayesian Source Apportionment Calculations of EC (Median, interquartile range $\left(25^{\text {th }}-75^{\text {th }}\right.$ percentile), and $95 \%$ Credible Intervals).

\begin{tabular}{|c|c|c|c|c|c|c|}
\hline & Seasons & summer & autumn & winter $^{\mathrm{b}}$ & spring & annual $^{b}$ \\
\hline Biomass burning & median & 0.136 & 0.177 & 0.221 & 0.156 & 0.173 \\
\hline \multirow[t]{2}{*}{ (C3 plants) } & $25^{\text {th }}-75^{\text {th }}$ percentile & $(0.129-0.142)$ & $(0.16-0.197)$ & $(0.196-0.242)$ & $(0.153-0.159)$ & $(0.166-0.18)$ \\
\hline & $95 \%$ credible intervals & $(0.113-0.159)$ & $(0.117-0.245)$ & $(0.106-0.288)$ & $(0.145-0.167)$ & $(0.15-0.196)$ \\
\hline \multirow[t]{3}{*}{ coal combustion } & median & 0.147 & 0.323 & 0.644 & 0.251 & 0.328 \\
\hline & $25^{\text {th }}-75^{\text {th }}$ percentile & $(0.086-0.23)$ & $(0.221-0.436)$ & $(0.534-0.709)$ & $(0.167-0.346)$ & $(0.25-0.403)$ \\
\hline & $95 \%$ credible intervals & $(0.025-0.494)$ & $(0.061-0.673)$ & $(0.165-0.805)$ & $(0.055-0.56)$ & $(0.117-0.557)$ \\
\hline \multirow[t]{3}{*}{ liquid fossil } & median & 0.717 & 0.497 & 0.136 & 0.594 & 0.499 \\
\hline & $25^{\text {th }}-75^{\text {th }}$ percentile & $(0.633-0.778)$ & $(0.383-0.607)$ & $(0.076-0.245)$ & $(0.498-0.677)$ & $(0.423-0.578)$ \\
\hline & $95 \%$ credible intervals & $(0.365-0.842)$ & $(0.147-0.774)$ & $(0.022-0.61)$ & $(0.282-0.79)$ & $(0.269-0.712)$ \\
\hline
\end{tabular}

${ }^{a}$ Three main source categories were differentiated using this technique: C3 plants (e.g., wood and crop residue), coal and liquid fossil fuel (e.g., oil, diesel, and gasoline) combustion.

${ }^{b}$ the sample taken on Chinese New Year eve (25 January 2009) was excluded. 
Table S7. The contribution of C3 and C4 plants burning to EC from the MCMC4 results ${ }^{\mathrm{a}}$ (Median, interquartile range $\left(25^{\text {th }}-75^{\text {th }}\right.$ percentile), and 95\% Credible Intervals).

\begin{tabular}{|c|c|c|c|c|c|c|}
\hline & Seasons & summer & autumn & winter $^{\mathrm{c}}$ & spring & annual $^{c}$ \\
\hline Biomass burning ${ }^{b}$ & median & 0.099 & 0.123 & 0.088 & 0.113 & 0.123 \\
\hline \multirow[t]{2}{*}{ from C3 plants } & $25^{\text {th }}-75^{\text {th }}$ percentile & $(0.080-0.114)$ & $(0.094-0.149)$ & $(0.058-0.122)$ & $(0.092-0.129)$ & $(0.105-0.139)$ \\
\hline & $95 \%$ credible intervals & $(0.037-0.137)$ & $(0.041-0.199)$ & $(0.019-0.196)$ & $(0.045-0.149)$ & $(0.07-0.165)$ \\
\hline \multirow{3}{*}{$\begin{array}{l}\text { Biomass burning } \\
\text { from } \mathrm{C} 4 \text { plants }\end{array}$} & median & 0.035 & 0.051 & 0.152 & 0.042 & 0.05 \\
\hline & $25^{\text {th }}-75^{\text {th }}$ percentile & $(0.022-0.054)$ & $(0.033-0.074)$ & $(0.119-0.182)$ & $(0.027-0.063)$ & $(0.034-0.066)$ \\
\hline & $95 \%$ credible intervals & $(0.007-0.097)$ & $(0.012-0.128)$ & $(0.052-0.240)$ & $(0.010-0.11)$ & $(0.013-0.097)$ \\
\hline
\end{tabular}

aResults from the four-sources (C3 biomass, C4 biomass, coal and liquid fossil fuel) MCMC4 model.

${ }^{\mathrm{b}}$ Contribution of biomass burning is shown in Table 2 and done by a posteriori combination of PDF for C3 plants and that for C4 plants.

'Sample taken from Chinese New Year eve (25 January 2009) was excluded. 
Table S8. Stable carbon isotopes for aerosols in China.

\begin{tabular}{|c|c|c|c|c|c|c|c|c|}
\hline Location & & Site type & Sampling period & Seasons & PM fraction & $\delta^{13} \mathrm{COC}_{\mathrm{OC}}(\%)$ & $\delta^{13} \mathrm{C}_{\mathrm{EC}}(\%)$ & References \\
\hline \multirow{6}{*}{$\begin{array}{l}\text { North } \\
\text { China }\end{array}$} & Beijing, China & urban & Jan, 2013 & winter & $\mathrm{PM}_{2.5}$ & $-24.26 \pm 0.29$ & & Yan et al. (2017) \\
\hline & Beijing, China & urban & Feb, 2010 & winter & $\mathrm{PM}_{2.5}$ & & -25.1 to -24.2 & Chen et al. (2013) \\
\hline & North China Plain & urban & Jan, 2013 & winter & $\mathrm{PM}_{2.5}$ & & -24.3 to -23.3 & Andersson et al. (2015) \\
\hline & 7 cities in North China & urban & Jan 6-20, 2003 & winter & $\mathrm{PM}_{2.5}$ & -25.54 to -23.08 & -25.02 to -23.27 & Cao et al. (2011b) \\
\hline & Beijing, China & urban & June, 2013 & summer & $\mathrm{PM}_{2.5}$ & $-26.74 \pm 0.65$ & & Yan et al. (2017) \\
\hline & 7 cities in North China & urban & June 3-July 30, 2003 & summer & $\mathrm{PM}_{2.5}$ & -26.90 to -26.33 & -26.62 to -25.27 & Cao et al. (2011b) \\
\hline \multirow{9}{*}{$\begin{array}{l}\text { South } \\
\text { China }\end{array}$} & Hong Kong & urban & Nov 2000-Feb 2001 & winter & $\mathrm{PM}_{2.5}$ & $-26.9 \pm 0.6$ & $-25.6 \pm 0.1$ & Ho et al. (2006) \\
\hline & Shanghai, China & urban & Jan, 2010 & winter & $\mathrm{PM}_{2.5}$ & & -25.8 to -24.7 & Chen et al. (2013) \\
\hline & Xiamen, China & urban & Dec, 2009 & winter & $\mathrm{PM}_{2.5}$ & & -25.3 to -24.9 & Chen et al. (2013) \\
\hline & Pearl River Delta & urban & Jan, 2013 & winter & $\mathrm{PM}_{2.5}$ & & -26.7 to -25.7 & Andersson et al. (2015) \\
\hline & Yangtze River Delta & urban & Jan, 2013 & winter & $\mathrm{PM}_{2.5}$ & & -27.7 to -25 & Andersson et al. (2015) \\
\hline & 7 cities in South China & urban & Jan 6-20, 2003 & winter & $\mathrm{PM}_{2.5}$ & -26.62 to -25.79 & -26.10 to -25.33 & Cao et al. (2011b) \\
\hline & Shanghai, China & urban & Sept 1-20, 2009 & Autumn & $\mathrm{PM}_{2.5}$ & $-24.5 \pm 0.8$ & $-25.1 \pm 0.6$ & Cao et al. (2013) \\
\hline & Hong Kong & urban & June-August, 2001 & summer & $\mathrm{PM}_{2.5}$ & $-26.9 \pm 0.5$ & $-25.6 \pm 0.1$ & Ho et al. (2006) \\
\hline & 7 cities in South China & urban & June 3-July 30, 2003 & summer & $\mathrm{PM}_{2.5}$ & -26.74 to -25.29 & -26.63 to -25.41 & Cao et al. (2011b) \\
\hline North & Xi'an China & urban & & winter & $\mathrm{PM}_{2.5}$ & $-24.13 \pm 0.83$ & $-23.20 \pm 0.35$ & This study \\
\hline \multirow[t]{4}{*}{ China } & & & & autumn & $\mathrm{PM}_{2.5}$ & $-24.85 \pm 0.79$ & $-25.14 \pm 0.66$ & \\
\hline & & & & summer & $\mathrm{PM}_{2.5}$ & $-25.73 \pm 0.90$ & $-25.94 \pm 0.46$ & \\
\hline & & & & spring & $\mathrm{PM}_{2.5}$ & $-26.58 \pm 0.57$ & $-25.40 \pm 0.33$ & \\
\hline & & & & annual & $\mathrm{PM}_{2.5}$ & $-25.32 \pm 1.19$ & $-24.92 \pm 1.14$ & \\
\hline
\end{tabular}


Table S9. Published OC/EC ratios for vehicle emissions specific to China.

\begin{tabular}{llll}
\hline OC/EC ratios & Sampling site & Measurement protocol & References \\
\hline $0.6 \pm 0.2$ & Shing Mun tunnel (Hongkong, China) & IMPROVE $^{\mathrm{a}}$ & Cheng et al. (2010) \\
$0.8 \pm 0.1$ & roadside sites (Hongkong, China) & IMPROVE $^{\mathrm{a}}$ & Cheng et al. (2010) \\
$0.86^{*}(0.48-1.45)^{* *}$ & Wuzushan tunnel (Yantai, China) & IMPROVE $^{\mathrm{a}}$ & Cui et al. (2016) \\
$1.03^{*}(0.77-1.35)^{* *}$ & Zhujiang tunnel (Guangzhou, China) & NIOSH $^{\mathrm{b}}$ & Dai et al. (2015) \\
$0.49 \pm 0.04$ & Zhujiang tunnel (Guangzhou, China) & NIOSH $^{\mathrm{b}}$ & He et al. (2008) \\
$(0.44-0.57)^{* *}$ & Zhujiang tunnel (Guangzhou, China) & NIOSH $^{\mathrm{b}}$ & Huang et al. (2006) \\
0.57 & Zhujiang tunnel (Guangzhou, China) & NIOSH $^{\mathrm{b}}$ & Zhang et al. (2015a) \\
\hline 1.45 & & &
\end{tabular}

*represents averaged values;

** represents the range of values;

${ }^{a}$ Chow et al. (2004);

${ }^{\mathrm{b}}$ Birch and Cary, (1996). 


\section{References}

Andersson, A., Deng, J., Du, K., Zheng, M., Yan, C., Sköld, M., and Gustafsson, Ö.: Regionallyvarying combustion sources of the January 2013 severe haze events over eastern China, Environ. Sci. Technol., 49, 2038-2043, 2015.

Birch, M. and Cary, R.: Elemental carbon-based method for monitoring occupational exposures to particulate diesel exhaust, Aerosol Sci. Technol., 25, 221-241, 1996.

Cao, G., Zhang, X., and Zheng, F.: Inventory of black carbon and organic carbon emissions from China, Atmos. Environ., 40, 6516-6527, 2006.

Cao, G., Zhang, X., Gong, S., An, X., and Wang, Y.: Emission inventories of primary particles and pollutant gases for China, Chin. Sci. Bull., 56, 781-788, $2011 \mathrm{a}$.

Cao, J. J., Chow, J. C., Tao, J., Lee, S.C., Watson, J. G., Ho, K.F., Wang, G.H., Zhu, C.S., and Han, Y.M.: Stable carbon isotopes in aerosols from Chinese cities: influence of fossil fuels, Atmos. Environ., 45, 1359-1363, 2011b.

Cao, J. J., Zhu, C. S., Tie, X. X., Geng, F. H., Xu, H. M., Ho, S. S. H., Wang, G. H., Han, Y. M., and Ho, K. F.: Characteristics and sources of carbonaceous aerosols from Shanghai, China, Atmos. Chem. Phys., 13, 803-817, 2013.

Chen, B., Andersson, A., Lee, M., Kirillova, E. N., Xiao, Q., Kruså, M., Shi, M., Hu, K., Lu, Z., Streets, D. G., Du, K., and Gustafsson, Ö.: Source forensics of black carbon aerosols from China, Environ. Sci. Technol., 47, 9102-9108, 2013.

Chen, Y., Sheng, G., Bi, X., Feng, Y., Mai, B., and Fu, J.: Emission factors for carbonaceous particles and polycyclic aromatic hydrocarbons from residential coal combustion in China, Environ. Sci. Technol., 39, 1861-1867, 2005.

Chen, Y., Zhi, G., Feng, Y., Fu, J., Feng, J., Sheng, G., and Simoneit, B. R. T.: Measurements of emission factors for primary carbonaceous particles from residential raw-coal combustion in China, Geophys. Res. Lett., 33, 2006.

Chen, Y., Cai, W., Huang, G., Li, J., and Zhang, G.:Stable carbon isotope of black carbon from typical emission sources in China. Environ. Sci. 33, 673-678, 2012 (in Chinese).

Chen, Y., Tian, C., Feng, Y., Zhi, G., Li, J., and Zhang, G.: Measurements of emission factors of PM2.5, OC, EC, and BC for household stoves of coal combustion in China, Atmos. Environ., 109, 190-196, 2015.

Cheng, Y., Lee, S. C., Ho, K. F., Chow, J. C., Watson, J. G., Louie, P. K. K., Cao, J. J., and Hai, X.: Chemically-speciated on-road $\mathrm{PM}_{2.5}$ motor vehicle emission factors in Hong Kong, Sci. Total Environ., 408, 1621-1627, 2010.

Chow, J. C., Watson, J. G., Crow, D., Lowenthal, D. H., and Merrifield, T.: Comparison of IMPROVE and NIOSH carbon measurements, Aerosol Sci. Technol., 34, 23-34, 2001.

Chow, J. C., Watson, J. G., Chen, L.-W. A., Arnott, W. P., Moosmüller, H., and Fung, K. K.: Equivalence of elemental carbon by Thermal/Optical Reflectance and Transmittance with different temperature protocols, Environ. Sci. Technol., 38, 4414-4422, 2004.

Chow, J. C., Watson, J. G., Chen, L.-W. A., Chang, M. O., Robinson, N. F., Trimble, D., and Kohl, S.: The IMPROVE_A temperature protocol for thermal/optical carbon analysis: maintaining consistency with a long-term database, J. Air Waste Manage., 57, 1014-1023, 2007.

Cui, M., Chen, Y., Tian, C., Zhang, F., Yan, C., and Zheng, M.: Chemical composition of PM 2.5 from two tunnels with different vehicular fleet characteristics, Sci. Total Environ., 550, 123-132, 2016.

Dai, S., Bi, X., Chan, L., He, J., Wang, B., Wang, X., Peng, P., Sheng, G., and Fu, J.: Chemical and stable carbon isotopic composition of $\mathrm{PM}_{2.5}$ from on-road vehicle emissions in the $\mathrm{PRD}$ region and implications for vehicle emission control policy, Atmos. Chem. Phys., 15, 3097-3108, 2015.

Das, O., Wang, Y., and Hsieh, Y.-P.: Chemical and carbon isotopic characteristics of ash and smoke derived from burning of C3 and C4 grasses, Org. Geochem., 41, 263-269, 2010. 
Guo, Z., Jiang, W., Chen, S., Sun, D., Shi, L., Zeng, G., and Rui, M.: Stable isotopic compositions of elemental carbon in $\mathrm{PM}_{1.1}$ in north suburb of Nanjing region, China, Atmos. Res., 168, 105-111, 2016.

Han, Y., Chen, L.-W., Huang, R.-J., Chow, J., Watson, J., Ni, H., Liu, S., Fung, K., Shen, Z., and Wei, C.: Carbonaceous aerosols in megacity Xi'an, China: Implications of thermal/optical protocols comparison, Atmos. Environ., 132, 58-68, 2016.

He, L.-Y., Hu, M., Zhang, Y.-H., Huang, X.-F., and Yao, T.-T.: Fine particle emissions from on-road vehicles in the Zhujiang Tunnel, China, Environ. Sci. Technol., 42, 4461-4466, 2008.

Ho, K., Lee, S., Cao, J., Li, Y., Chow, J. C., Watson, J. G., and Fung, K.: Variability of organic and elemental carbon, water soluble organic carbon, and isotopes in Hong Kong, Atmos. Chem. Phys., 6, 4569-4576, 2006.

Huang, X. F., Yu, J. Z., He, L. Y., and Hu, M.: Size distribution characteristics of elemental carbon emitted from Chinese vehicles: Results of a tunnel study and atmospheric implications, Environ. Sci. Technol., 40, 5355-5360, 2006.

Kawashima, H. and Haneishi, Y.: Effects of combustion emissions from the Eurasian continent in winter on seasonal $\delta^{13} \mathrm{C}$ of elemental carbon in aerosols in Japan, Atmos. Environ., 46, 568-579, 2012.

Li, J., Wang, G., Ren, Y., Wang, J., Wu, C., Han, Y., Zhang, L., Cheng, C., and Meng, J.: Identification of chemical compositions and sources of atmospheric aerosols in Xi'an, inland China during two types of haze events, Sci. Total Environ., 566, 230-237, 2016a.

Li, Q., Li, X., Jiang, J., Duan, L., Ge, S., Zhang, Q., Deng, J., Wang, S., and Hao, J.: Semi-coke briquettes: towards reducing emissions of primary $\mathrm{PM}_{2.5}$, particulate carbon, and carbon monoxide from household coal combustion in China, Sci. Rep., 6, 2016b.

Liu, G., Li, J., Xu, H., Wu, D., Liu, Y., and Yang, H.: Isotopic compositions of elemental carbon in smoke and ash derived from crop straw combustion, Atmos. Environ., 92, 303-308, 2014.

Martinelli, L., Camargo, P., Lara, L., Victoria, R., and Artaxo, P.: Stable carbon and nitrogen isotopic composition of bulk aerosol particles in a C4 plant landscape of southeast Brazil, Atmos. Environ., 36, 2427-2432, 2002.

Ni, H. Y., Han, Y. M., Cao, J. J., Chen, L.-W. A., Tian, J., Wang, X. L., Chow, J. C., Watson, J. G., Wang, Q. Y., Wang, P., Li, H., and Huang, R. J.: Emission characteristics of carbonaceous particles and trace gases from open burning of crop residue in China, Atmos. Environ., 123, 399-406, 2015.

Qin, Y. and Xie, S.: Historical estimation of carbonaceous aerosol emissions from biomass open burning in China for the period 1990-2005, Environ. Pollut., 159, 3323, 2011.

Shen, G., Yang, Y., Wang, W., Tao, S., Zhu, C., Min, Y., Xue, M., Ding, J., Wang, B., Wang, R., Shen, H., Li, W., Wang, X., and Russell, A. G.: Emission factors of particulate matter and elemental carbon for crop residues and coals burned in typical household stoves in China, Environ. Sci. Technol., 44, 7157-7162, 2010.

Shen, G., Chen, Y., Xue, C., Lin, N., Huang, Y., Shen, H., wang, Y., Li, T., Zhang, Y., and Su, S.: Pollutant emissions from improved coal-and wood-fuelled cookstoves in rural households, Environ. Sci. Technol., 49, 6590-6598, 2015.

Streets, D., Yarber, K., Woo, J. H., and Carmichael, G.: Biomass burning in Asia: Annual and seasonal estimates and atmospheric emissions, Global Biogeochem. Cy., 17, 2003.

Tian, J., Ni, H., Cao, J., Han, Y., Wang, Q., Wang, X., Chen, L. W. A., Chow, J. C., Watson, J. G., Wei, C., Sun, J., Zhang, T., and Huang, R.: Characteristics of carbonaceous particles from residential coal combustion and agricultural biomass burning in China, Atmos. Pollut. Res., 8, 521-527, 2017.

Yan, C., Zheng, M., Bosch, C., Andersson, A., Desyaterik, Y., Sullivan, A. P., Collett, J. L., Zhao, B., Wang, S., and He, K.: Important fossil source contribution to brown carbon in Beijing during winter, Sci. Rep., 7, 2017.

Yan, X., Ohara, T., and Akimoto, H.: Bottom-up estimate of biomass burning in mainland China, Atmos. Environ., 40, 5262-5273, 2006. 
Zhang, H., Wang, S., Hao, J., Wan, L., Jiang, J., Zhang, M., Mestl, H. E. S., Alnes, L. W. H., Aunan, K., and Mellouki, A. W.: Chemical and size characterization of particles emitted from the burning of coal and wood in rural households in Guizhou, China, Atmos. Environ., 51, 94-99, 2012.

Zhang, Q., Klimont, Z., Streets, D. G., Huo, H., and He, K. B.: An anthropogenic PM emission model for China and emission inventory for the year 2001, Prog. Nat. Sci., 16, 223-231, 2006 (in Chinese).

Zhang, Q., Streets, D. G., Carmichael, G. R., He, K., Huo, H., Kannari, A., Klimont, Z., Park, I., Reddy, S., and Fu, J.: Asian emissions in 2006 for the NASA INTEX-B mission, Atmos. Chem. Phys., 9, 5131-5153, 2009.

Zhang, T., Cao, J. J., Tie, X. X., Shen, Z. X., Liu, S. X., Ding, H., Han, Y. M., Wang, G. H., Ho, K. F., Qiang, J., and Li, W. T.: Water-soluble ions in atmospheric aerosols measured in Xi'an, China: seasonal variations and sources, Atmos. Res., 102, 110-119, 2011.

Zhang, Y. L., Li, J., Zhang, G., Zotter, P., Huang, R.-J., Tang, J.-H., Wacker, L., Prévôt, A. S. H., and Szidat, S.: Radiocarbon-based source apportionment of carbonaceous aerosols at a regional background site on Hainan island, South China, Environ. Sci. Technol., 48, 2651-2659, 2014.

Zhang, Y. L., Wang, X., Li, G., Yang, W., Huang, Z., Zhang, Z., Huang, X., Deng, W., Liu, T., Huang, Z., and Zhang, Z.: Emission factors of fine particles, carbonaceous aerosols and traces gases from road vehicles: Recent tests in an urban tunnel in the Pearl River Delta, China, Atmos. Environ., 122, 876$884,2015 a$.

Zhang, Y. L., Huang, R. J., El Haddad, I., Ho, K. F., Cao, J. J., Han, Y., Zotter, P., Bozzetti, C., Daellenbach, K. R., Canonaco, F., Slowik, J. G., Salazar, G., Schwikowski, M., Schnelle-Kreis, J., Abbaszade, G., Zimmermann, R., Baltensperger, U., Prévôt, A. S. H., and Szidat, S.: Fossil vs. nonfossil sources of fine carbonaceous aerosols in four Chinese cities during the extreme winter haze episode of 2013, Atmos. Chem. Phys., 15, 1299-1312, $2015 \mathrm{~b}$.

Zhang, Y. X., Schauer, J. J., Zhang, Y., Zeng, L., Wei, Y., Liu, Y., and Shao, M.: Characteristics of particulate carbon emissions from real-world Chinese coal combustion, Environ. Sci. Technol., 42, 5068-5073, 2008.

Zhang, Y. S., Shao, M., Lin, Y., Luan, S., Mao, N., Chen, W., and Wang, M.: Emission inventory of carbonaceous pollutants from biomass burning in the Pearl River Delta Region, China, Atmos. Environ., 76, 189-199, 2013.

Zhi, G., Chen, Y., Feng, Y., Xiong, S., Li, J., Zhang, G., Sheng, G., and Fu, J.: Emission characteristics of carbonaceous particles from various residential coal-stoves in China, Environ. Sci. Technol., 42, 3310-3315, 2008.

Zhou, Y., Xing, X., Lang, J., Chen, D., Cheng, S., Wei, L., Wei, X., and Liu, C.: A comprehensive biomass burning emission inventory with high spatial and temporal resolution in China, Atmos. Chem. Phys., 17, 2839-2864, 2017. 